\title{
Accessibility of nonlinear time-delay systems
}

\author{
Claudia Califano*, Claude H. Moog**
}

\begin{abstract}
A full characterization of accessibility is provided for nonlinear time-delay systems. It generalizes the rank condition which is known for weak controllability of linear timedelay systems, as well as the celebrated geometric approach for delay-free nonlinear systems and the characterization of their accessibility. Besides, fundamental results are derived on integrability and basis completion which are of major importance for a number of general control problems for nonlinear timedelay systems. They are shown to impact preconceived ideas about canonical forms for nonlinear time-delay systems.
\end{abstract}

Index Terms - Time-delay systems; accessibility; autonomous element; geometric approach; nonlinear systems.

\section{INTRODUCTION}

Time-delay systems are modeled by ordinary differential equations which involve delayed variables [9], [16] and are typically encountered in biology or biomedical systems [25], in telerobotics, teleoperations [12], [15] and in networked control systems. Unfortunately, the theory for such systems is much less developed than it is for linear time-delay systems. Even fundamental properties such as accessibility or observability and related design problems are far from being understood.

A sufficient condition for accessibility of nonlinear timedelay systems can be found in [18]. Whether this condition is necessary remains an open problem. Among the contributions in this paper, a full characterization of accessibility is derived in terms of a necessary and sufficient rank condition for nonlinear time-delay systems. This result is in the continuation of the celebrated geometric approach for delay-free systems; the work [10] on accessibility has certainly been the seminal paper inspiring the geometric approach that started to be developed by Lobry, Jurdjevic, Sussmann, Hermes, Krener, Sontag, Brockett in the early 1970's (quoted from [23]).

Herein it is also proven that any nonlinear time-delay system can always be decomposed into a non accessible subsystem and a fully accessible one by means of a bicausal state transformation. This is far from being obvious as such a decomposition does not always exist with respect to observability, as displayed in the following example. Consider

$$
\begin{aligned}
\dot{x}_{i} & =0, \quad i=1,2 \\
y & =x_{1}(t) x_{1}(t-1)+x_{2}(t) x_{2}(t-1) .
\end{aligned}
$$

As any time-derivative of the output is zero, for $t \geq 0$, the two state variables of the above system can not be estimated independently and the system is not fully observable. From

\footnotetext{
*C.Califano is with Dipartimento di Ingegneria Informatica Automatica e Gestionale Antonio Ruberti, Università di Roma La Sapienza, Via Ariosto 25,00185 Italy; califano@dis . uniroma1. it

** C.H. Moog is with LUNAM, IRCCyN, UMR C.N.R.S. 6597, 1 rue de la Noë, BP 92101, 44321 Nantes Cedex 3, France; moog@ieee.org
}

the results of this paper, there is no invertible change of state coordinates which decomposes the system into an observable subsystem and a non observable one. This contradicts common beliefs on this matter. Additional assumptions are required [27] to ensure that such a decomposition still exists.

The results in the paper feature fundamentals of a novel approach to tackle nonlinear time-delay systems. They include useful algebraic results which are independent of any system dynamics. A basis completion theorem is obtained which may impact future research on time-delay systems. From above, given a set of causal exact one-forms, it is not always possible to find additional causal exact one-forms to define a unimodular matrix. Necessary and sufficient conditions are derived under which such a transformation exists.

A major difficulty in analyzing time-delay systems is their infinite dimensionality. Thus, in the nonlinear case, integrability results provided by Poincaré Lemma or Frobenius Theorem have to be revised. A sequence of finite dimensional systems is introduced and shown to capture major structural properties of time-delay systems. Standard tools on those finite dimensional systems become efficient and circumvent this difficulty.

The outline of the paper is as follows. This introductory section is ended up with a summary of the main results which are put into perspective with respect to control systems. Section II introduces general notations about the class of dynamical systems which are considered. Mathematical tools adapted for infinite dimensional systems are introduced and results on integrability are derived in Section III. Section IV includes the characterization of accessibility and the corresponding decomposition of systems. Two examples are provided in Section V to illustrate the approach of the paper: the JAKSTAT signaling pathway Model borrowed from biology, and the Chained Form Model used in Mechanical Engineering. Concluding remarks are found in Section VI.

\section{SUMMARY OF THE CONTRIBUTIONS}

The main original contributions given in this paper are summarized hereafter. Some of them implicate received ideas.

\section{Integrability}

The integrability problem of a submodule was addressed in [2]. The new contributions in that respect are detailed in Section III as follows:

- A new notion of Polynomial Lie Bracket is introduced in Section III, which allows to give necessary and sufficient conditions for the integrability of a given submodule, stated in the framework of polynomial modules. 
- Corollary 1 gives an upper bound on the maximum delay which characterizes these exact differentials after integration. An algorithm for the computation of a basis over $\mathcal{K}(\delta]$ for such exact differentials is included, without using any Taylor expansion and thus reducing the computational complexity.

- Theorem 3 solves the integrability problem in the most general case whereas in [2] the results were restricted to the special case of causal right submodules $^{1}$. Note that causal exact forms, may have a non causal right annihilator as it is the case for instance for $\omega=d\left(x_{1}(t) x_{1}(t-1)+x_{2}(t) x_{2}(t-1)\right)$.

- It is shown that the exact differentials which characterize the left annihilator of the given submodule can be computed by referring to a finite dimensional distribution of proper dimension.

- Theorem 2 in Section III fully characterizes those closed bases of exact differentials which can be completed to get a bicausal change of coordinates, generalizing preliminary results given in [5]. This last result represents an important milestone for the study of nonlinear delay systems since it is not valid in general. A typical counterexample is given again by $\omega$ defined above.

\section{Characterization of Accessibility}

Theorem 5 in Section IV displays the rank condition which generalizes the well established full dimensional condition of the strong accessibility distribution for delay-free nonlinear systems. It also somehow generalizes the Kalman criterion for the study of controllability of linear time-invariant systems.

\section{Decomposition with respect to Accessibility}

It was easily shown that the decomposition with respect to observability does not exist for a general nonlinear time-delay system. Theorem 6 shows that the decomposition with respect to accessibility is always possible.

\section{NOTATIONS}

Consider the class of nonlinear time-delay systems

$$
\begin{aligned}
& \dot{x}(t)=F(x(t), x(t-D), \cdots, x(t-s D))+ \\
& \left.\sum_{i=0}^{l} \sum_{j=1}^{m} G_{j i}(x(t), x(t-D), \cdots, x(t-s D)) u_{j}(t-i D)\right),
\end{aligned}
$$

where $D$ is a constant delay, $s, l \geq 0$ are integers and the functions $G_{j i}(x(t), \cdots, x(t-s D)), j \in[1, m], i \in[0, l]$ and $F(x(t), \cdots, x(t-s D))$ are analytic in their arguments. Such a class of systems covers the case of constant multiple commensurate delays as well [9].

General notations valid throughout the paper are as follows.

- $\mathbf{x}_{[p, s]}^{T}=\left(x^{T}(t+p D), \cdots x^{T}(t-s D)\right) \in \mathbb{R}^{(p+s+1) n}$, denotes the vector consisting of the np future values

\footnotetext{
${ }^{1}$ A submodule is causal if its generators are causal, that is they do not depend on $t+i, i>0$.
}

$x(t+i D), i \in[1, p]$, of the state together with the first $(s+1) n$ components of the state of the infinite dimensional system (1). When $p=0$, the more simple notation $\mathbf{x}_{[s]}^{T}=\mathbf{x}_{[0, s]}^{T} \in \mathbb{R}^{(s+1) n}$ is used, with $\mathbf{x}_{[0]}=\left[x_{1,[0]}, \cdots, x_{n,[0]}\right]^{T}=x(t) \in \mathbb{R}^{n}, \mathbf{u}_{[0]}=$ $\left[u_{1,[0]}, \cdots, u_{m,[0]}\right]^{T}=u(t) \in \mathbb{R}^{m}$, the current values of the state and input variables.

- $\mathbf{x}_{[p, s]}^{T}(-i)=\left(x^{T}(t+p D-i D), \cdots x^{T}(t-s D-i D)\right)$. Accordingly, $\mathbf{x}_{[s]}(-i)=\mathbf{x}_{[0, s]}(-i) ; x_{j,[0]}(-i):=x_{j}(t-$ $i D)$, and $u_{\ell,[0]}(-i):=u_{\ell}(t-i D)$ denote respectively the $j$-th and $\ell$-th components of the current values of the state and input variables delayed by $\tau=i D$. When no confusion is possible the subindex will be omitted so that $\mathbf{x}$ will stand for $\mathbf{x}_{[p, s]}$, while $\mathbf{x}(-i)$ for $\mathbf{x}_{[p, s]}(-i)$.

- $\mathbf{u}^{[j]}:=\left(\mathbf{u}^{T}, \dot{\mathbf{u}}^{T}, \cdots,\left(\mathbf{u}^{(j)}\right)^{T}\right)^{T}$ where $\mathbf{u}^{[-1]}=\emptyset$;

- $\mathcal{K}^{*}$ denotes the field of meromorphic functions $f\left(\mathbf{x}_{[p, s]}, \mathbf{u}_{[q, j]}^{[k]}\right)$, with $p, s, k, q, j \in \mathbb{N}$. The subfield $\mathcal{K}$ of $\mathcal{K}^{*}$, consisting of causal meromorphic functions, is obtained for $p=q=0$.

- Given a function $f\left(\mathbf{x}_{[p, s]}, \mathbf{u}_{[q, j]}^{[k]}\right)$, we will denote by $f(-l)=f\left(\mathbf{x}_{[p, s]}(-l), \mathbf{u}_{[q, j]}^{[k]}(-l)\right)$;

- $d$ is the standard differential operator;

- $\delta$ represents the backward time-shift operator: for $a(\cdot), f(\cdot) \in \mathcal{K}^{*}: \delta[a d f]=a(-1) \delta d f=a(-1) d f(-1) ;$

- $\mathcal{K}^{*}(\delta]$ is the (left) ring of polynomials in $\delta$ with coefficients in $\mathcal{K}^{*}$. Every element of $\mathcal{K}^{*}(\delta]$ may be written as $\alpha(\delta]=\sum_{j=0}^{r_{\alpha}} \alpha_{j}(\cdot) \delta^{j}$, with $\alpha_{j}(\cdot) \in \mathcal{K}^{*}$ and $r_{\alpha}=\operatorname{deg}(\alpha(\delta])$ the polynomial degree in $\delta$. Let $\beta(\delta]=$ $\sum_{j=0}^{r_{\beta}} \beta_{j}(\cdot) \delta^{j}$ be an element of $\mathcal{K}^{*}(\delta]$ of polynomial degree $r_{\beta}$. Then addition and multiplication on this ring are defined by ([26]): $\alpha(\delta]+\beta(\delta]=\sum_{i=0}^{\max \left\{r_{\alpha}, r_{\beta}\right\}}\left(\alpha_{i}+\beta_{i}\right) \delta^{i}$ and $\alpha(\delta] \beta(\delta]=\sum_{i=0}^{r_{\alpha}} \sum_{j=0}^{r_{\beta}} \alpha_{i} \beta_{j}(-i) \delta^{i+j}$.

Analogously $\mathcal{K}(\delta]$ is the (left) ring of polynomials in $\delta$ with coefficients in $\mathcal{K}$.

- Let for $i \in[1, j], \tau_{i}\left(\mathbf{x}_{[l]}\right)$ be vector fields defined in an open set $\Omega_{l} \subseteq \mathbb{R}^{n(l+1)}$. Then $\Delta=\operatorname{span}\left\{\tau_{i}\left(\mathbf{x}_{[l]}\right), i=\right.$ $1, \ldots, j\}$ represents the distribution generated by the vector fields $\tau_{i}(\cdot)$ and defined on $\mathbb{R}^{n(l+1)} \cdot \bar{\Delta}$ represents its involutive closure, that is, for any two vector fields $\tau_{i}(\cdot), \tau_{j}(\cdot) \in \bar{\Delta}$ then also the Lie bracket $\left[\tau_{i}, \tau_{j}\right]=$ $\frac{\partial \tau_{i}}{\partial \mathbf{x}_{[l]}} \tau_{j}-\frac{\partial \tau_{j}}{\partial \mathbf{x}_{[l]}} \tau_{i} \in \bar{\Delta}([11])$.

$\Delta_{[p, q]} \quad$ will denote a distribution in $\operatorname{span}_{\mathcal{K} *}\left\{\frac{\partial}{\partial \mathbf{x}_{[0]}(p)}, \cdots, \frac{\partial}{\partial \mathbf{x}_{[0]}(-q)}\right\}$.

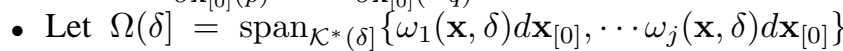
be a left submodule of rank $j$ with $\omega_{i} \in \mathcal{K}^{*(1 \times n)}(\delta]$. Any $\omega(\mathbf{x}, \delta) d \mathbf{x}_{[0]} \in \Omega(\delta]$ can be expressed as $\omega(\mathbf{x}, \delta) d \mathbf{x}_{[0]}=\sum_{i=1}^{j} \alpha_{i}(\mathbf{x}, \delta) \omega_{i}(\mathbf{x}, \delta) d \mathbf{x}_{[0]}$. The left closure of $\Omega(\delta]$ is the largest left submodule $\Omega_{c}(\delta]$ of rank $j$ containing $\Omega(\delta]$ ([6]). Analogously let $\Delta(\delta]=$ $\operatorname{span}_{\mathcal{K}^{*}(\delta]}\left\{\tau_{1}(\mathbf{x}, \delta), \cdots \tau_{j}(\mathbf{x}, \delta)\right\}$ be a right submodule of rank $j$ with $\tau_{i} \in \mathcal{K}^{*(n \times 1)}(\delta]$. Any $\tau(\mathbf{x}, \delta) \in \Delta(\delta]$ can be expressed as $\tau(\mathbf{x}, \delta)=\sum_{i=1}^{j} \tau_{i}(\mathbf{x}, \delta) \alpha_{i}(\mathbf{x}, \delta)$. The right closure of $\Delta(\delta]$ is the largest right submodule $\Delta_{c}(\delta]$ of rank $j$ containing $\Delta(\delta]$. When no confusion is possible $\mathcal{K}^{* n}(\delta]$ will be used at the place of $\mathcal{K}^{*(n \times 1)}(\delta]$. 


\section{RESULTS ON INTEGRABILITY}

Consider the right submodule

$$
\Delta(\delta]=\operatorname{span}_{\mathcal{K}^{*}(\delta]}\left\{r_{1}(\mathbf{x}, \delta), \cdots, r_{j}(\mathbf{x}, \delta)\right\}
$$

of rank $j$, with the polynomial vector $r_{i}(\mathbf{x}, \delta)=$ $\sum_{\ell=0}^{\bar{s}}\left(r_{i}^{\ell}(\mathbf{x})\right)^{T} \frac{\partial}{\partial \mathbf{x}_{[0]}(p)} \delta^{\ell} \in \mathcal{K}^{* n}(\delta]$. By assumption $r_{i}^{\bar{s}+\ell}=0$, $\forall \ell>0$; by convention $r_{i}^{-\ell}=0, \forall \ell>0$.

Integrating $\Delta(\delta]$ consists in the computation of a set of $n-j$ exact differentials $d \lambda_{\mu}(\mathbf{x})=\Lambda_{\mu}(\mathbf{x}, \delta) d \mathbf{x}_{[0]}(p)$ independent over $\mathcal{K}^{*}(\delta]$, which define a basis for the left kernel of $\Delta(\delta]$.

Definition 1: The right submodule $\Delta(\delta]$ of rank $j$, given by (2), is $p$-integrable if there exist $n-j$ independent exact differentials $d \lambda_{\mu}(\mathbf{x})=\Lambda_{\mu}(\mathbf{x}, \delta) d \mathbf{x}_{[0]}(p), \mu \in[1, n-j]$ such that $d \lambda_{\mu}(\mathbf{x})=\Lambda_{\mu}(\mathbf{x}, \delta) d \mathbf{x}_{[0]}(p)$ lay in the left kernel of $\Delta(\delta]$, that is $d \lambda_{\mu}(\mathbf{x}) r_{i}(\mathbf{x}, \delta)=0$, for $i \in[1, j]$ and $\mu \in[1, n-j]$, and any other exact differential $d \bar{\lambda}(\mathbf{x}) \in \Delta^{\perp}(\delta]$, can be expressed as linear combination over $\mathcal{K}^{*}(\delta]$ of such $d \lambda_{\mu}(\mathbf{x})$ 's.

Definition 2: The right submodule $\Delta(\delta]$ of rank $j$, given by (2), is said to be integrable if there exists some finite integer $p$ such that $\Delta(\delta]$ is $p$-integrable.

Example 1: Consider for instance

$$
\Delta(\delta]=\operatorname{span}_{\mathcal{K}^{*}(\delta]}\left\{\left(\begin{array}{c}
-x_{1}(2) \delta \\
x_{2}(2)
\end{array}\right)\right\}
$$

According to the above definition, $\Delta(\delta]$ is 2-integrable, since $d \lambda=d\left(x_{1}(2) x_{2}(1)\right)=\left(x_{2}(1), x_{1}(2) \delta\right) d x(2) \perp \Delta(\delta]$. How to check the existence of such a solution and how to compute it, is the topic of the present Section.

To this end, the definitions of Generalized Lie derivative, Generalized Lie Bracket ${ }^{2}$ (different definitions can be found in [8], [22]), Involutivity and Involutive Closure of a right submodule are introduced next. They represent the nontrivial generalization of the standard definitions used in the delayfree context, which can be recovered as a special case. These definitions play a fundamental role in the integrability conditions.

\section{A. Generalized Lie Derivative and Generalized Lie Bracket}

Definition 3: Given the function $\tau\left(\mathbf{x}_{[p, s]}\right)$ and the submodule element $r(\mathbf{x}, \delta)=\sum_{j=0}^{\bar{s}} r^{j}(\mathbf{x}) \delta^{j} \in \mathcal{K}^{* n}(\delta]$, the Generalized Lie derivative $L_{r^{\mu}(\mathbf{x})} \tau\left(\mathbf{x}_{[p, s]}\right)$ is defined as

$$
L_{r^{\mu}(\mathbf{x})} \tau\left(\mathbf{x}_{[p, s]}\right)=\sum_{l=-p}^{\mu} \frac{\partial \tau\left(\mathbf{x}_{[p, s]}\right)}{\partial \mathbf{x}_{[0]}(-l)} r^{\mu-l}(\mathbf{x}(-l)) .
$$

Definition 4: Let $r_{q}(\mathbf{x}, \delta)=\sum_{j=0}^{\bar{s}} r_{q}^{j}(\mathbf{x}) \delta^{j} \in \mathcal{K}^{* n}(\delta]$, $q=1,2$. For any $k, l \geq 0$, the Generalized Lie bracket

${ }^{2}$ The definitions of Extended Lie derivative and Extended Lie bracket given in [2], [3] are recovered as a special case when the considered functions and vectors are causal. $\left[r_{1}^{k}(\cdot), r_{2}^{l}(\cdot)\right]_{E_{i}}$, on $\mathbb{R}^{(i+1) n}, i \geq 0$, is defined as

$$
\left[r_{1}^{k}(\cdot), r_{2}^{l}(\cdot)\right]_{E_{i}}=\sum_{j=0}^{i}\left(\left[r_{1}^{k-j}, r_{2}^{l-j}\right]_{E}\right)_{(\mathbf{x}(-j))}^{T} \frac{\partial}{\partial \mathbf{x}_{[0]}(-j)}
$$

where

$$
\left[r_{1}^{k}(\cdot), r_{2}^{l}(\cdot)\right]_{E}=\left(L_{r_{1}^{k}(\mathbf{x})} r_{2}^{l}(\mathbf{x})-L_{r_{2}^{l}(\mathbf{x})} r_{1}^{k}(\mathbf{x})\right) .
$$

Remark. The Generalized Lie derivative as defined by (3) is the Lie derivative of $\tau\left(\mathbf{x}_{[p, s]}\right)$ along

$$
\left(r^{\mu+p}(+p), \cdots, r^{\mu}(0), r^{\mu-1}(-1), \cdots, r^{0}(-\mu), 0\right)^{T} .
$$

The latter is embedded in

$$
\Delta_{[p, q]}=\operatorname{span}_{\mathcal{K}^{*}}\left(\begin{array}{ccccc}
\mathbf{r}^{0}(\mathbf{x}(p)) & \cdots & \mathbf{r}^{\ell}(\mathbf{x}(p)) & 0 & 0 \\
0 & \ddots & & \ddots & 0 \\
0 & 0 & \mathbf{r}^{0}(\mathbf{x}(-q)) & \cdots & \mathbf{r}^{\ell}(\mathbf{x}(-q))
\end{array}\right) .
$$

where $\mathbf{r}^{i}(\mathbf{x})=\left(r_{1}^{i}, \cdots, r_{j}^{i}\right)$ and $q>\mu$. Accordingly, assuming without loss of generality $k \geq l$, the Generalized Lie bracket $\left[r_{1}^{k}(\cdot), r_{2}^{l}(\cdot)\right]_{E_{i}}$, is defined starting from the standard Lie Bracket

$$
\left[\left(\begin{array}{c}
0 \\
r_{1}^{s}(s-k) \\
\vdots \\
r_{1}^{k}(0) \\
\vdots \\
\vdots \\
r_{1}^{0}(-k) \\
0
\end{array}\right)\left(\begin{array}{c}
r_{2}^{s}(s-l) \\
\vdots \\
\vdots \\
r_{2}^{l}(0) \\
\vdots \\
r_{2}^{0}(-l) \\
0 \\
0
\end{array}\right)\right]=\left(\begin{array}{c}
\tau^{k+s-l}(s-l) \\
\vdots \\
\vdots \\
\tau^{k}(0) \\
\vdots \\
\vdots \\
\tau^{0}(-k) \\
0
\end{array}\right)
$$

In fact, $\left[r_{1}^{k}(\cdot), r_{2}^{l}(\cdot)\right]_{E_{i}}=\sum_{j=0}^{\min (k, i)}\left(\tau^{k-j}(-j)\right)^{T} \frac{\partial}{\partial \mathbf{x}_{[0]}(-j)} \cdot \triangleleft$

The Generalized Lie brackets (4) are associated to $\Delta_{[p, q]}$ defined above. In the special case of causal submodules (which lead to consider $\Delta_{[0, q]}$, they have shown to characterize the 0 -integrability conditions, that is when the $\Delta^{\perp}(\delta]$ is generated by $d \lambda_{\mu}(\mathbf{x})=\Lambda_{\mu}(\mathbf{x}, \delta) d \mathbf{x}_{[0]}, \mu \in[1, n-j]$ [2]. However, if we refer to the submodule $\Delta(\delta]$ given by (2), there is no condition expressed in this framework. To overcome this problem, the following definition of polynomial Lie bracket is required and a more general definition of Lie bracket is also introduced.

Definition 5: Given $r_{i}\left(\mathbf{x}_{\left[s_{i}, s\right]}, \delta\right) \in \mathcal{K}^{* n}(\delta], i=1,2$, the Lie Bracket $\left[r_{1}\left(\mathbf{x}_{\left[s_{1}, s\right]}, \delta\right), r_{2}\left(\mathbf{x}_{\left[s_{2}, s\right]}, \delta\right)\right]$, is a $\left(4 s+s_{1}+s_{2}+1\right)$ uple of polynomial vectors $r_{12, j}(\mathbf{x}, \delta)$, defined as

$r_{12, j}(\mathbf{x}, \delta)=\sum_{\ell=-s_{1}}^{2 s+s_{1}}\left[r_{1}^{\ell+s_{1}-j}, r_{2}^{\ell}\right]_{E_{0}} \delta^{\ell+s_{1}}, j \in\left[-2 s, 2 s+s_{1}+s_{2}\right]$.

Recalling that a polynomial vector $r_{1}\left(\mathbf{x}_{\left[s_{i}, s\right]}, \delta\right)$ acts on a function $\epsilon(t)$ and denoting its image as $\mathbf{R}_{1}\left(\mathbf{x}_{\left[s_{1}, s\right]}, \epsilon\right):=$ $\sum_{j=0}^{s} r_{1}^{j}(\mathbf{x}) \epsilon(-j)$, the Polynomial Lie Bracket is then defined as follows: 
Definition 6: Given $r_{i}\left(\mathbf{x}_{\left[s_{i}, s\right]}, \delta\right) \in \mathcal{K}^{* n}(\delta], i=1,2$, the Polynomial Lie Bracket $\left[\mathbf{R}_{1}(\mathbf{x}, \epsilon), r_{2}(\mathbf{x}, \delta)\right]$ is defined as

$$
\begin{gathered}
{\left[\mathbf{R}_{1}(\mathbf{x}, \epsilon), r_{2}(\mathbf{x}, \delta)\right]:=a d_{\mathbf{R}_{1}\left(\mathbf{x}_{\left[s_{1}, s\right]}, \epsilon\right)} r_{2}\left(\mathbf{x}_{\left[s_{2}, s\right]}, \delta\right)=} \\
\left.\dot{r}_{2}(\mathbf{x}, \delta)\right|_{\dot{x}_{[0]}=\mathbf{R}_{1}(\mathbf{x}, \epsilon)} \delta^{s_{1}}-\sum_{k=0}^{s_{1}+s} \frac{\partial \mathbf{R}_{1}\left(\mathbf{x}_{\left[s_{1}, s\right]}, \epsilon\right)}{\partial \mathbf{x}_{[0]}\left(s_{1}-k\right)} \delta^{k} r_{2}\left(\mathbf{x}\left(s_{1}\right), \delta\right) .
\end{gathered}
$$

With some abuse, the Polynomial Lie Bracket and the standard Lie bracket are both denoted by [., .]. No confusion is possible, since in the Polynomial Lie bracket, some $\epsilon(i)$ will always be present inside the brackets.

\section{Some Remarks:}

- The link between the Lie bracket (6) and the Generalized Lie bracket (4) can be easily established by noting that setting $\mathbf{I}(\delta)=\left(\begin{array}{llll}I_{n} \delta^{2\left(s+s_{1}\right)}, & \cdots, \quad I_{n} \delta, & I_{n}\end{array}\right)$

$r_{12, j}(\mathbf{x}, \delta)=\mathbf{I}(\delta)\left(\left.\left[r_{1}^{2\left(s+s_{1}\right)-j}, r_{2}^{2 s+s_{1}}\right]_{E_{2 s+s_{1}}}\right|_{\mathbf{x}\left(2\left(s+s_{1}\right)\right)}\right)$

- Standard computations on the Polynomial Lie Bracket show that

$$
\left[\mathbf{R}_{1}(\mathbf{x}, \epsilon), r_{2}(\mathbf{x}, \delta)\right]=\sum_{j=-2 s}^{2 s+s_{1}+s_{2}} r_{12, j}(\mathbf{x}, \delta) \epsilon(j) .
$$

- If the given vectors are independent of $\delta$ and of the delay, one recovers (up to $\epsilon(0)$ ), the standard Lie bracket since

$$
\left[\mathbf{R}_{1}(\mathbf{x}, \epsilon), r_{2}(\mathbf{x}, \delta)\right]=\left[r_{1}^{0}(x) \epsilon(0), r_{2}^{0}(x)\right]=\left[r_{1}^{0}, r_{2}^{0}\right] \epsilon(0) .
$$

Instead, if delays are present, $\left[\mathbf{R}_{1}(\mathbf{x}, \epsilon), r_{2}(\mathbf{x}, \delta)\right]$ immediately enlightens some important differences with respect to the delay-free case, such as the loss of validity of the Straightening Theorem. In fact, since the term depending on $\delta$ undergoes a different kind of operation with respect to the term depending on $\epsilon$, starting from $r(\mathbf{x}, \delta)$ and its corresponding image $\mathbf{R}(\mathbf{x}, \epsilon)$, in general

$\left.\dot{r}(\mathbf{x}, \delta)\right|_{\dot{x}_{[0]}=\mathbf{R}(\mathbf{x}, \epsilon)} \delta^{s_{1}} \neq \sum_{k=0}^{s_{1}+s} \frac{\partial \mathbf{R}\left(\mathbf{x}_{\left[s_{1}, s\right]}, \epsilon\right)}{\partial \mathbf{x}_{[0]}\left(s_{1}-k\right)} \delta^{k} r\left(\mathbf{x}\left(s_{1}\right), \delta\right)$

which yields that in general $[r(\mathbf{x}, \delta), r(\mathbf{x}, \delta)] \neq 0$. For instance, consider $r(\mathbf{x}, \delta)=\left(\begin{array}{c}x_{2}(-1) \\ 1\end{array}\right)$. Then $\mathbf{R}(\mathbf{x}, \epsilon)=\left(\begin{array}{c}x_{2}(-1) \\ 1\end{array}\right) \epsilon(0)$ and

$$
[\mathbf{R}(\mathbf{x}, \epsilon), r(\mathbf{x}, \delta)]=\left(\begin{array}{c}
\epsilon(-1)-\epsilon(0) \delta \\
0
\end{array}\right) \neq 0 .
$$

Accordingly

$$
[r(\mathbf{x}, \delta), r(\mathbf{x}, \delta)]=\left\{\left(\begin{array}{l}
1 \\
0
\end{array}\right),\left(\begin{array}{c}
-\delta \\
0
\end{array}\right)\right\} .
$$

The Polynomial Lie Bracket $\left[\mathbf{R}_{1}(\mathbf{x}, \epsilon), r_{2}(\mathbf{x}, \delta)\right]$ has the following properties:

Proposition 1 (Anticommutativity): Assume without loss of generality, $s_{2} \geq s_{1}$, then for any integer $j$,

$$
\frac{\partial\left[\mathbf{R}_{1}(\mathbf{x}, \epsilon), r_{2}(\mathbf{x}, \delta)\right]}{\partial \epsilon\left(s_{1}-j\right)} \delta^{s_{2}-s_{1}+j+|j|}=-\frac{\partial\left[\mathbf{R}_{2}(\mathbf{x}, \epsilon), r_{1}(\mathbf{x}, \delta)\right]}{\partial \epsilon\left(s_{2}+j\right)} \delta^{|j|}(8)
$$

Proposition 2: Given for $i=1,2, \bar{r}_{i}\left(\mathbf{x}_{\left[\bar{s}_{i}, s\right]}, \delta\right)=$ $r_{i}\left(\mathbf{x}_{\left[s_{i}, s\right]}, \delta\right) \beta_{i}\left(\mathbf{x}_{\left[s_{i}, s\right]}, \delta\right)$, then

$$
\left[\overline{\mathbf{R}}_{1}(\mathbf{x}, \epsilon), \bar{r}_{2}(\mathbf{x}, \delta)\right] \delta^{s_{1}-\bar{s}_{1}}=
$$

$\left[\mathbf{R}_{1}(\mathbf{x}, \bar{\epsilon}), r_{2}(\mathbf{x}, \delta)\right]_{\bar{\epsilon}=\beta_{1}(\mathbf{x}, \epsilon)} \hat{\beta}_{2}+r_{2}(\mathbf{x}, \delta) \alpha_{2}-r_{1}(\mathbf{x}, \delta) \alpha_{1}$

with $\hat{\beta}_{2}=\beta_{2}\left(\mathbf{x}\left(s_{1}\right), \delta\right), \alpha_{1}=\sum_{k=0}^{s+s_{1}} \frac{\partial \beta_{1}(\mathbf{x}, \epsilon)}{\partial \mathbf{x}_{[0]}\left(s_{1}-k\right)} \delta^{k} \bar{r}_{2}\left(\mathbf{x}\left(s_{1}\right), \delta\right)$, and $\alpha_{2}=\left.\dot{\beta}_{2}(\mathbf{x}, \delta)\right|_{\dot{x}=\overline{\mathbf{R}}_{1}(\mathbf{x}, \epsilon)} \delta^{s_{1}}$.

Remark. While the proofs are reported in the Appendix, it is worth pointing out that the standard properties of Lie brackets for delay-free systems are recovered. In fact, if $r_{i}(\mathbf{x}, \delta)=$ $r_{i}^{0}(x)$, for $i=1,2$, then $\mathbf{R}_{i}(\mathbf{x}, \epsilon)=r_{i}^{0}(x) \epsilon(0)$ and

$$
\begin{aligned}
\frac{\partial\left[\mathbf{R}_{1}(\mathbf{x}, \epsilon), r_{2}(\mathbf{x}, \delta)\right]}{\partial \epsilon(0)} & =\left[r_{1}^{0}, r_{2}^{0}\right]=-\left[r_{2}^{0}, r_{1}^{0}\right] \\
& =-\frac{\partial\left[\mathbf{R}_{2}(\mathbf{x}, \epsilon), r_{1}(\mathbf{x}, \delta)\right]}{\partial \epsilon(0)}
\end{aligned}
$$

whereas letting $\bar{r}_{i}(\mathbf{x}, \delta)=r_{i}^{0}(x) \beta_{i}(x)$, then $\overline{\mathbf{R}}_{i}(\mathbf{x}, \epsilon)=$ $r_{i}^{0}(x) \beta_{i}(x) \epsilon(0)$ and

$$
\begin{aligned}
{\left[\overline{\mathbf{R}}_{1}(\mathbf{x}, \epsilon), \bar{r}_{2}(\mathbf{x}, \delta)\right] } & =\left[r_{1}^{0}(x) \beta_{1}(x) \epsilon(0), r_{2}^{0}(x) \beta_{2}(x)\right] \\
& =\left(\left[r_{1}^{0}, r_{2}^{0}\right] \beta_{2} \beta_{1}+r_{2}^{0} \alpha_{2}-r_{1}^{0} \alpha_{1}\right) \epsilon(0)
\end{aligned}
$$

with $\alpha_{1}=\beta_{2}\left(L_{r_{2}^{0}} \beta_{1}\right)$ and $\alpha_{2}=\beta_{1}\left(L_{r_{1}^{0}} \beta_{2}\right) . \triangleleft$

Example 2: Consider for $i=1,2, r_{i}(\mathbf{x}, \delta)$ given by

$$
r_{1}(\mathbf{x}, \delta)=\left(\begin{array}{c}
x_{1}(1) \\
x_{2} \delta
\end{array}\right), \quad r_{2}(\mathbf{x}, \delta)=\left(\begin{array}{c}
x_{2} \delta \\
x_{1}
\end{array}\right) .
$$

Then

$$
\mathbf{R}_{1}(\mathbf{x}, \epsilon)=\left(\begin{array}{c}
x_{1}(1) \epsilon(0) \\
x_{2} \epsilon(-1)
\end{array}\right), \quad \mathbf{R}_{2}(\mathbf{x}, \epsilon)=\left(\begin{array}{c}
x_{2} \epsilon(-1) \\
x_{1} \epsilon(0)
\end{array}\right) .
$$

Accordingly, since $s_{1}=1, s_{2}=s=0$,

$$
\begin{gathered}
{\left[\mathbf{R}_{1}(\mathbf{x}, \epsilon), r_{2}(\mathbf{x}, \delta)\right]=\left(\begin{array}{c}
x_{2} \epsilon(-1) \delta \\
x_{1}(1) \epsilon(0)
\end{array}\right) \delta-\left(\begin{array}{c}
\epsilon(0) x_{2}(1) \delta \\
\epsilon(-1) x_{1} \delta
\end{array}\right)} \\
=-\left(\begin{array}{c}
0 \\
x_{1} \delta
\end{array}\right) \epsilon(0)+\left(\begin{array}{c}
x_{2} \delta^{2}-x_{2}(1) \delta \\
x_{1}(1) \delta
\end{array}\right) \epsilon(1) \\
=r_{12,0}(\mathbf{x}, \delta) \epsilon(0)+r_{12,1}(\mathbf{x}, \delta) \epsilon(1)
\end{gathered}
$$

One can easily verify that

$$
\begin{aligned}
& r_{12,0}(\mathbf{x}, \delta)=-\left(\begin{array}{c}
0 \\
x_{1}
\end{array}\right) \delta=\sum_{\ell=-1}^{1}\left[r_{1}^{\ell+1}, r_{2}^{\ell}\right]_{E_{0}} \delta^{\ell+1} \\
& r_{12,1}(\mathbf{x}, \delta)=\left(\begin{array}{c}
-x_{2}(1) \\
x_{1}(1)
\end{array}\right) \delta+\left(\begin{array}{c}
x_{2} \\
0
\end{array}\right) \delta^{2}=\sum_{\ell=-1}^{1}\left[r_{1}^{\ell}, r_{2}^{\ell}\right]_{E_{0}} \delta^{\ell+1},
\end{aligned}
$$

which confirms (6).

$$
\text { Analogously, }\left[\mathbf{R}_{2}(\mathbf{x}, \epsilon), r_{1}(\mathbf{x}, \delta)\right]=\left(\begin{array}{c}
x_{2}(1)-x_{2} \delta \\
-x_{1}(1)
\end{array}\right) \epsilon(0)+
$$
$\left(\begin{array}{c}0 \\ x_{1} \delta\end{array}\right) \epsilon(1)$ and it is again easily verified that (8) holds true 
(with the indices exchanged since $s_{1}>s_{2}$ ). In fact,

$\begin{aligned} \frac{\partial\left[\mathbf{R}_{2}(\mathbf{x}, \epsilon), r_{1}(\mathbf{x}, \delta)\right]}{\partial \epsilon(0)} \delta & =\left(\begin{array}{c}x_{2}(1)-x_{2} \delta \\ -x_{1}(1)\end{array}\right) \delta \\ & =-\frac{\partial\left[\mathbf{R}_{1}(\mathbf{x}, \epsilon), r_{2}(\mathbf{x}, \delta)\right]}{\partial \epsilon(1)} \\ \frac{\partial\left[\mathbf{R}_{2}(\mathbf{x}, \epsilon), r_{1}(\mathbf{x}, \delta)\right]}{\partial \epsilon(1)} \delta & =\left(\begin{array}{c}0 \\ x_{1} \delta\end{array}\right) \delta=-\frac{\partial\left[\mathbf{R}_{1}(\mathbf{x}, \epsilon), r_{2}(\mathbf{x}, \delta)\right]}{\partial \epsilon(0)} \delta .\end{aligned}$

\section{B. Involutivity of a right submodule versus its Integrability}

The integrability of a left-submodule of one forms is sketched in [13] and worked out in [14]. For right submodules, to deal with integrability, the involutivity concept has to be defined.

Definition 7: Consider the right submodule

$$
\Delta(\delta]=\operatorname{span}_{\mathcal{K} *(\delta]}\left\{r_{1}(\mathbf{x}, \delta), \cdots, r_{j}(\mathbf{x}, \delta)\right\}
$$

of rank $j$, with $r_{i}(\mathbf{x}, \delta)=\sum_{l=0}^{s} r_{i}^{l}\left(\mathbf{x}_{\left[s_{i}, s\right]}\right) \delta^{l}$ and let $\Delta_{c}(\delta]$ be its right closure. Then $\Delta(\delta]$ is said to be involutive if for any pair of indices $i, \ell \in[1, j]$ the Lie Bracket $\left[r_{i}(\mathbf{x}, \delta), r_{\ell}(\mathbf{x}, \delta)\right]$ satisfies

$$
\operatorname{span}_{\mathcal{K}^{*}(\delta]}\left\{\left[r_{i}(\mathbf{x}, \delta), r_{\ell}(\mathbf{x}, \delta)\right]\right\} \subset \Delta_{c}(\delta]
$$

Remark. Definition 7 includes as a special case the notion of involutivity of a distribution. The main feature is that starting from a given right submodule, its involutivity implies that the Lie bracket of two of its elements can not be obtained as a linear combination of the generators of the given submodule, but it is a linear combination of the generators of its right closure. For finite dimensional systems, distributions are closed by definition, so there is no such a difference. $\triangleleft$

The definition of involutivity of a submodule is crucial for the integrability problem, as enlightened in the next theorem.

Theorem 1: The right submodule

$$
\Delta(\delta]=\operatorname{span}_{\mathcal{K}^{*}(\delta]}\left\{r_{1}(\mathbf{x}, \delta), \cdots, r_{j}(\mathbf{x}, \delta)\right\}
$$

of rank $j$, is completely 0-integrable if and only if it is involutive and its left annihilator is causal.

Proof. Necessity. Assume that there exist $n-j$ causal exact differentials $d \lambda_{i}(\mathbf{x})=\Lambda_{i}(\mathbf{x}, \delta) d \mathbf{x}_{[0]}$, independent over $\mathcal{K}^{*}(\delta]$ which are in $\Delta^{\perp}(\delta]$. Let $\rho$ denote the maximum between the delay in the state variable and the degree in $\delta$. Then

$$
\Lambda_{\mu}\left(\mathbf{x}_{[\rho]}, \delta\right) r_{\ell}(\mathbf{x}, \delta)=0, \forall \mu \in[1, n-j], \forall \ell \in[1, j]
$$

The time derivative of (11) along $\mathbf{R}_{q}\left(\mathbf{x}_{\left[s_{1}, s\right]}, \epsilon\right)$, yields $\forall \mu \in$ $[1, n-j], \forall \ell \in[1, j]$

$\left.\dot{\Lambda}_{\mu}(\mathbf{x}, \delta)\right|_{\dot{\mathbf{x}}_{[0]}=\mathbf{R}_{q}(\mathbf{x}, \epsilon)} r_{\ell}(\mathbf{x}, \delta)+\left.\Lambda_{\mu}(\mathbf{x}, \delta) \dot{r}_{\ell}(\mathbf{x}, \delta)\right|_{\dot{\mathbf{x}}_{[0]}=\mathbf{R}_{q}(\mathbf{x}, \epsilon)}=0$

Multiplying on the right by $\delta^{s_{1}}$ one gets

$$
\begin{gathered}
\sum_{i, k=0}^{\rho}\left(\frac{\partial\left(\Lambda_{\mu}^{i}(\mathbf{x})\right)^{T}}{\partial \mathbf{x}_{[0]}(-k)} \mathbf{R}_{q}(\mathbf{x}(-k), \epsilon(-k))\right)^{T} \delta^{i} r_{\ell}(\mathbf{x}, \delta) \delta^{s_{1}} \\
+\left.\Lambda_{\mu}(\mathbf{x}, \delta) \dot{r}_{\ell}(\mathbf{x}, \delta)\right|_{\dot{\mathbf{x}}_{[0]}=\mathbf{R}_{q}(\mathbf{x}, \epsilon)} \delta^{s_{1}}=0
\end{gathered}
$$

that is, recalling that $\frac{\partial\left(\Lambda_{\mu}^{i}(\mathbf{x})\right)^{T}}{\partial \mathbf{x}_{[0]}(-k)}=\left(\frac{\partial\left(\Lambda_{\mu}^{k}(\mathbf{x})\right)^{T}}{\partial \mathbf{x}_{[0]}(-i)}\right)^{T}$,

$$
\begin{gathered}
\sum_{i, k=0}^{\rho}\left(\left(\frac{\partial\left(\Lambda_{\mu}^{k}(\mathbf{x})\right)}{\partial \mathbf{x}_{[0]}(-i)}\right)^{T} \mathbf{R}_{q}(\mathbf{x}(-k), \epsilon(-k))\right)^{T} \delta^{i} r_{\ell}(\mathbf{x}, \delta) \delta^{s_{1}}+ \\
+\Lambda_{\mu}(\mathbf{x}, \delta) \sum_{k=0}^{s+s_{1}} \frac{\partial \mathbf{R}_{q}(\mathbf{x}, \epsilon)}{\partial \mathbf{x}_{[0]}\left(s_{1}-k\right)} \delta^{k} r_{\ell}\left(\mathbf{x}\left(s_{1}\right), \delta\right)= \\
-\Lambda_{\mu}(\mathbf{x}, \delta)\left[\mathbf{R}_{q}(\mathbf{x}, \epsilon), r_{\ell}(\mathbf{x}, \delta]\right.
\end{gathered}
$$

Moreover, since $\lambda_{\mu}(\mathbf{x})$ is causal then $\frac{\partial \Lambda_{\mu}^{k}(\mathbf{x})}{\partial \mathbf{x}_{[0]}\left(s_{1}-i\right)}=0$ for $i \in\left[0, s_{1}-1\right]$; since $\Lambda_{\mu}(\mathbf{x}, \delta) r_{q}(\mathbf{x}, \delta) \stackrel{=}{=}$, then also $\sum_{k=0}^{s} \Lambda_{\mu}^{k}(\mathbf{x}) \mathbf{R}_{q}(\mathbf{x}(-k), \epsilon(-k))=0$, so that for $i \in\left[0, s+s_{1}\right]$,

$$
\begin{aligned}
\sum_{k=0}^{s} \mathbf{R}_{q}^{T}(\mathbf{x}(-k), \epsilon(-k)) & \frac{\partial\left(\Lambda_{\mu}^{k}(\mathbf{x})\right)^{T}}{\partial \mathbf{x}_{[0]}(-i)}+ \\
& +\sum_{k=0}^{s} \Lambda_{\mu}^{k}(\mathbf{x}) \frac{\partial \mathbf{R}_{q}(\mathbf{x}(-k), \epsilon(-k))}{\partial \mathbf{x}_{[0]}(-i)}=0 .
\end{aligned}
$$

It follows, through standard computations, that

$$
\begin{gathered}
\sum_{i=0}^{s+s_{1}} \sum_{k=0}^{s}\left(\left(\frac{\partial\left(\Lambda_{\mu}^{k}(\mathbf{x})\right)^{T}}{\partial \mathbf{x}_{[0]}\left(s_{1}-i\right)}\right)^{T} \mathbf{R}_{q}(\mathbf{x}(-k), \epsilon(-k))\right)^{T} \delta^{i}= \\
-\sum_{i=0}^{s+s_{1}} \Lambda_{\mu}(\mathbf{x}, \delta) \frac{\partial \mathbf{R}_{q}(\mathbf{x}, \epsilon)}{\partial \mathbf{x}_{[0]}\left(s_{1}-i\right)} \delta^{i}
\end{gathered}
$$

which, substituted in (12), leads to

$$
\Lambda_{\mu}(\mathbf{x}, \delta)\left[\mathbf{R}_{q}(\mathbf{x}, \epsilon), r_{\ell}(\mathbf{x}, \delta)\right]=0, \quad \forall \epsilon .
$$

Since the previous relation has to be satisfied $\forall \mu \in[1, n-j]$, and $\forall \ell, q \in[1, j]$, then necessarily $\Delta(\delta]$ is involutive.

Sufficiency. Let $\omega\left(\mathbf{x}_{[\hat{s}]}, \delta\right)=\left(\omega_{1}^{T}\left(\mathbf{x}_{[\hat{s}]}, \delta\right), \cdots, \omega_{n-j}^{T}\left(\mathbf{x}_{[\hat{s}]}, \delta\right)\right)^{T}$ be the left annihilator of $\left(r_{1}\left(\mathbf{x}_{\left[s_{1}, s\right]}, \delta\right), \cdots, r_{j}\left(\mathbf{x}_{\left[s_{k}, s\right]}, \delta\right)\right)$. Let $\bar{s}=\max \left\{s_{1}, \cdots, s_{k}\right\}$ and $\rho=\max \{\hat{s}, \operatorname{deg}(\omega(\mathbf{x}, \delta))\}$, that is, for $k \in[1, n-j], \omega_{k}(\mathbf{x}, \delta)=\sum_{\ell=0}^{\rho} \omega_{k}^{\ell}\left(\mathbf{x}_{[\rho]}\right) \delta^{\ell}$. Set $\Omega=\left(0, \cdots, 0, \omega^{0}\left(\mathbf{x}_{[\rho]}\right), \cdots, \omega^{\rho}\left(\mathbf{x}_{[\rho]}\right), 0, \cdots, 0\right)$, where $\omega^{0}$ is preceded by $\bar{s}$ 0- blocks, and set $\Delta_{i}:=\Delta_{[\bar{s}, i+s]} \subset$ $\operatorname{span}\left\{\frac{\partial}{\partial \mathbf{x}_{[0]}(\bar{s})}, \cdots, \frac{\partial}{\partial \mathbf{x}_{[0]}(-i-s)}\right\}$ as

$$
\Delta_{i}=\operatorname{span}_{\mathcal{K}^{*}}\left(\begin{array}{ccccccc}
I_{n \bar{s}} & * & * & 0 & \cdots & & 0 \\
0 & \mathbf{r}^{0}(\mathbf{x}) & \cdots & \mathbf{r}^{\ell}(\mathbf{x}) & 0 & & \\
\vdots & 0 & \ddots & & \ddots & \ddots & \\
& \vdots & 0 & \mathbf{r}^{0}(\mathbf{x}(-i)) & \cdots & \mathbf{r}^{\ell}(\mathbf{x}(-i)) & 0 \\
0 & \cdots & 0 & \cdots & 0 & I_{n s}
\end{array}\right)
$$

By assumption $\omega(\mathbf{x}, \delta)$ is causal and for any two vector fields $\tau_{q} \in \Delta_{i 0}, q=1,2, i \geq \rho, \Omega \tau_{q}=0$ and $\Omega\left[\tau_{1}, \tau_{2}\right]=0$. Moreover, since $i \geq \rho, \Omega \frac{\partial}{\partial x_{\ell,[0]}(-i-p)}=0, \forall \ell \in[1, n]$, $\forall p \in[1, s]$. It follows that $\Omega\left[\tau_{1}, \frac{\partial}{\partial x_{\ell,[0]}(-i-p)}\right]=0$, since $\frac{\partial\left(\Omega \tau_{1}\right)}{\partial x_{\ell,[0]}(-i-p)}=\Omega \frac{\partial \tau_{1}}{\partial x_{\ell,[0]}(-i-p)}=0$. Analogously, since $\Omega$ is causal, then for any $p \in[1, \bar{s}], \frac{\partial\left(\Omega \tau_{1}\right)}{\partial x_{\ell,[0]}(+p)}=\Omega \frac{\partial \tau_{1}}{\partial x_{\ell,[0]}(+p)}=0$, which shows that $\Omega\left[\tau_{1}, \frac{\partial}{\partial x_{\ell,[0]}(+p)}\right]=0$, so that $\Omega \perp \bar{\Delta}_{i}$. As a consequence, there exist at least $n-j$ causal exact differentials, independent over $\mathcal{K}^{*}$ which lay in the left annihilator of $\bar{\Delta}_{i}$. 
It remains to show that there are also $n-j$ causal exact differentials, independent over $\mathcal{K}^{*}(\delta]$, which lay in the left annihilator of $\Delta(\delta]$. This follows immediately by noting that if $d \lambda_{1}, \cdots, d \lambda_{\mu}, \mu \leq n-j$, is a basis for $\Delta^{\perp}(\delta]$, then $\omega(\mathbf{x}, \delta) d \mathbf{x}_{[0]}=\sum_{i=1}^{\mu} \alpha_{i}(\mathbf{x}, \delta) d \lambda_{i}$ since $\Omega$ is 0 -integrable. Since the $\omega_{i}(\mathbf{x}, \delta) d \mathbf{x}_{[0]}$ 's are $n-j$ and by assumption they are independent over $\mathcal{K}^{*}(\delta]$, then necessarily $\mu=n-j . \triangleleft$ A direct consequence of the proof of Theorem 1 is the definition of an upper bound on the maximum delay appearing in the exact differentials which generate a basis for the left annihilator of $\Delta(\delta]$. This is pointed out in the next corollary.

Corollary 1: Let the right submodule

$$
\Delta(\delta]=\operatorname{span}_{\mathcal{K}^{*}(\delta]}\left\{r_{1}(\mathbf{x}, \delta), \cdots, r_{j}(\mathbf{x}, \delta)\right\}
$$

of rank $j$, with $r_{i}(\mathbf{x}, \delta)=\sum_{l=0}^{\bar{s}} r_{i}^{l}\left(\mathbf{x}_{\left[s_{i}, s\right]}\right) \delta^{l}$, be completely 0 -integrable. Then the maximum delay which characterizes the exact differentials which generate the left annihilator of $\Delta(\delta]$ is not greater than $j \bar{s}+s$.

Proof. The proof of Theorem 1 shows that if $\rho$ is the maximum between the degree in $\delta$ and the largest delay affecting the state variables in the left annihilator $\Omega\left(\mathbf{x}_{[\bar{p}]}, \delta\right)$ of $\Delta(\delta]$, then the exact differentials are affected by a maximum delay which is not greater than $\rho$. According to Lemma $4, \operatorname{deg}(\Omega(\mathbf{x}, \delta)) \leq j \bar{s}$, whereas $\bar{p} \leq s+j \bar{s}$, which shows that $\rho \leq j \bar{s}+s$.

The result stated by Theorem 1, which is itself an important achievement, plays also a key role in proving a series of fundamental results which are enlightened hereafter.

1) Bicausal change of coordinates: As already noticed in the Introduction, a major problem in control theory stands in the possibility of describing the given system in some different coordinates which may put in evidence particular structural properties. In the delay context it is fundamental to be able to compute bicausal change of coordinates, that is, diffeomorphisms which are causal and admit a causal inverse and which are defined as follows:

Definition 8: Consider a system $\Sigma$ in the state coordinates $x$. The mapping $\mathbf{z}_{[0]}=\varphi\left(\mathbf{x}_{[\alpha]}\right)$, where $\alpha \in \mathbb{N}$ and $\varphi \in$ $\mathcal{K}^{n}$, is a local bicausal change of coordinates for $\Sigma$ if there exists an integer $\ell \in \mathbb{N}$ and a function $\psi\left(\mathbf{z}_{[\ell]}\right) \in \mathcal{K}^{n}$ such that, assuming $\mathbf{z}_{[0]}$ and $\mathbf{x}_{[0]}$ defined for $t \geq-(\alpha+\ell)$, then $\psi\left(\varphi\left(\mathbf{x}_{[\alpha]}\right), \cdots, \varphi\left(\mathbf{x}_{[\alpha]}(-\ell)\right)\right)=\mathbf{x}_{[0]}$ for $t \geq 0$.

The next result completely characterizes such a class of change of coordinates.

Theorem 2: Given $k$ functions $\lambda_{i}\left(\mathbf{x}_{[\alpha]}\right), i \in[1, k]$, whose differentials are independent over $\mathcal{K}(\delta]$, there exist $n-k$ functions $\theta_{j}\left(\mathbf{x}_{[\bar{\alpha}]}\right), j \in[1, n-k]$ such that $\operatorname{span}_{\mathcal{K}(\delta]}\left\{d \lambda_{1}, \cdots, d \lambda_{k}, d \theta_{1}, \cdots, d \theta_{n-k}\right\} \equiv \operatorname{span}_{\mathcal{K}(\delta]}\left\{d \mathbf{x}_{[0]}\right\}$ if and only if $\operatorname{span}_{\mathcal{K}(\delta]}\left\{d \lambda_{1}, \cdots, d \lambda_{k}\right\}$ is closed and its right annihilator is causal. As a consequence $d \mathbf{z}_{[0]}=$ $\left(d \lambda_{1}^{T}, \cdots, d \lambda_{k}^{T}, d \theta_{1}^{T}, \cdots, d \theta_{n-k}^{T}\right)^{T}$ defines a bicausal change of coordinates.

Proof. If the $k$ exact differentials $d \lambda_{i}(\mathbf{x})$ can be completed to span all $d \mathbf{x}_{[0]}$ over $\mathcal{K}(\delta]$ then necessarily
$\operatorname{span}_{\mathcal{K}(\delta]}\left\{d \lambda_{1}, \cdots, d \lambda_{k}\right\}$ must be closed and its right annihilator must be causal. On the contrary, due to Lemma 3 in the Appendix, if $\operatorname{span}_{\mathcal{K}(\delta]}\left\{d \lambda_{1}, \cdots, d \lambda_{k}\right\}$ is closed and its right annihilator is causal then one can compute an exact differential $d \theta_{1}$ independent over $\mathcal{K}(\delta]$ of the $d \lambda_{i}$ 's and such that $\operatorname{span}_{\mathcal{K}(\delta]}\left\{d \lambda_{1}, \cdots, d \lambda_{k}, d \theta_{1}\right\}$ is closed and its right annihilator is causal. Iterating, one gets the result. $\triangleleft$

2) p-integrability: The approach presented in this paper allows us to state a more general result concerning $p$ integrability. This is done hereafter.

Theorem 3: The right submodule

$$
\Delta(\delta]=\operatorname{span}_{\mathcal{K}^{*}(\delta]}\left\{r_{1}(\mathbf{x}, \delta), \cdots, r_{j}(\mathbf{x}, \delta)\right\}
$$

of rank $j$, is completely $p$-integrable if and only if

$\hat{\Delta}(\delta]=\Delta(\mathbf{x}(-p), \delta)=\operatorname{span}_{\mathcal{K}^{*}(\delta]}\left\{r_{1}(\mathbf{x}(-p), \delta), \cdots, r_{j}(\mathbf{x}(-p), \delta)\right\}$

is completely 0 -integrable.

Proof. Assume that $\Delta(\delta]$ is completely $p$-integrable. Then there exist $n-j$ independent exact differentials $d \lambda_{i}(\mathbf{x})=$ $\Lambda_{i}(\mathbf{x}, \delta) d \mathbf{x}_{[0]}(p)$ such that $\Lambda(\mathbf{x}, \delta) \Delta(\delta]=0$, with $\Lambda(\mathbf{x}, \delta)=$ $\left(\Lambda_{1}^{T}(\mathbf{x}, \delta), \cdots, \Lambda_{n-j}^{T}(\mathbf{x}, \delta)\right)^{T}$. Consequently, for $i \in[1, j]$,

$$
\delta^{p} \Lambda(\mathbf{x}, \delta) r_{i}(\mathbf{x}, \delta)=\Lambda(\mathbf{x}(-p), \delta) r_{i}(\mathbf{x}(-p), \delta) \delta^{p}=0,
$$

that is $\Lambda(\mathbf{x}(-p), \delta) \hat{\Delta}(\delta]=0$. Noting that $\delta^{p} \Lambda(\mathbf{x}, \delta) d \mathbf{x}_{[0]}(p)=$ $\Lambda(\mathbf{x}(-p), \delta) d \mathbf{x}_{[0]}$ proves that $\hat{\Delta}(\delta]$ is 0 -integrable. Conversely, if $\hat{\Delta}(\delta]$ is 0 -integrable, there exist $n-j$ exact differentials $d \bar{\lambda}_{i}(\mathbf{x})=\bar{\Lambda}_{i}(\mathbf{x}, \delta) d \mathbf{x}_{[0]}$ such that $\bar{\Lambda}(\mathbf{x}, \delta) \hat{\Delta}(\delta]=0$. As a consequence also $\bar{\Lambda}(\mathbf{x}, \delta) \hat{\Delta}(\delta] \delta^{p}=0$, which shows that $\hat{\Delta}(\mathbf{x}(p), \delta)=\Delta(\delta]$ is $p$-integrable. $\triangleleft$

3) Smallest 0-integrable right submodule containing $\Delta(\delta]$ : If the given submodule $\Delta(\delta]$ is not 0 -integrable, one may be interested in computing the smallest 0 -integrable right submodule containing it. The following definition needs to be introduced, which generalizes the notion of involutive closure of a distribution to the present context.

Definition 9: Given the right submodule

$$
\Delta(\delta]=\operatorname{span}_{\mathcal{K}^{*}(\delta]}\left\{r_{1}(\mathbf{x}, \delta), \cdots, r_{j}(\mathbf{x}, \delta)\right\}
$$

of rank $j$, with $r_{i}(\mathbf{x}, \delta)=\sum_{l=0}^{s} r_{i}^{l}\left(\mathbf{x}_{\left[s_{i}, s\right]}\right) \delta^{l}$, let $\Delta_{c}(\delta]$ be its right closure. Then its involutive closure $\bar{\Delta}(\delta]$ is the smallest submodule, which contains $\Delta_{c}(\delta]$ and which is involutive.

Accordingly, the following result can be stated.

Theorem 4: Consider the right submodule

$$
\Delta(\delta]=\operatorname{span}_{\mathcal{K}^{*}(\delta]}\left\{r_{1}(\mathbf{x}, \delta), \cdots, r_{j}(\mathbf{x}, \delta)\right\}
$$

of rank $j$, and let $\bar{\Delta}(\delta]$ be its involutive closure and assume that the left annihilator of $\bar{\Delta}(\delta]$ is causal. Then $\bar{\Delta}(\delta]$ is the smallest completely 0 -integrable right submodule containing $\Delta(\delta]$. 


\section{ACCESSIBILITY OF NONLINEAR TIME-DELAY SYSTEMS}

In this Section, the accessibility properties of the given system are fully characterized in terms of absence of non constant autonomous functions. Using an algebraic terminology, the latter reduces to the accessibility module $\mathcal{R}_{n}$ introduced in [18] and defined by (21) to be torsion free over the ring $\mathcal{K}(\delta]$. This has been worked out in [7] for the special case of linear time-delay systems.

Within the framework of this paper, the following definition of accessibility is stated.

Definition 10: A system is fully accessible if there doesn't exist any autonomous function for the system, that is a non constant function $\lambda(\mathbf{x})$ whose time derivative of any order along the dynamics of the system, is never affected by the control.

Example 3: Consider the delay-free second order nonlinear system $\dot{x}_{1}(t)=x_{2}(t) u(t), \dot{x}_{2}(t)=u(t)$ (chained form). It is well known that such a system is not locally accessible. The accessibility distribution associated to it is $\mathcal{R}_{2}=\operatorname{span}\left(\begin{array}{c}x_{2} \\ 1\end{array}\right)$, which has dimension 1 for any $x$. As a matter of fact, the function $\varphi=x_{1}(t)-\frac{1}{2} x_{2}^{2}(t)$ is an autonomous function for the given system and it is computed starting from $\mathcal{R}_{2}$. Introducing a delay on $x_{2}$ renders the system locally accessible, as shown in [4] for the nonlinear system $\dot{x}_{1}(t)=x_{2}(t-1) u(t)$, $\dot{x}_{2}(t)=u(t)$. This is discussed in Example 4. Using the results obtained in this Section, it is shown that the rank of the accessibility submodule associated to a given delay system, determines the dimension of its accessible subsystem and consequently that of its non accessible part.

To this end, consider system (1), which, using the notation introduced in Section II, reads

$$
\dot{\mathbf{x}}_{[0]}=F\left(\mathbf{x}_{[s]}\right)+\sum_{i=0}^{l} \sum_{j=1}^{m} G_{j i}\left(\mathbf{x}_{[s]}\right) u_{j,[0]}(-i)
$$

By applying the differential operator $d$ to both sides of (14), one gets its differential form representation given by

$$
d \dot{\mathbf{x}}_{[0]}=f\left(\mathbf{x}_{[s]}, \mathbf{u}_{[0]}, \delta\right) d \mathbf{x}_{[0]}+\mathbf{g}_{1}\left(\mathbf{x}_{[s]}, \delta\right) d \mathbf{u}_{[0]},
$$

where

$$
\begin{aligned}
& f(\mathbf{x}, \mathbf{u}, \delta)=\sum_{i=0}^{s}\left(\frac{\partial F(\mathbf{x})}{\partial \mathbf{x}_{[0]}(-i)}+\sum_{j=1}^{m} \sum_{k=0}^{l} u_{j,[0]}(-k) \frac{\partial G_{j k}(\mathbf{x})}{\partial \mathbf{x}_{[0]}(-i)}\right) \delta^{i},(16) \\
& \mathbf{g}_{1}(\mathbf{x}, \delta)=\left(g_{11}, \cdots, g_{1 m}\right), g_{1 i}=\sum_{k=0}^{l} G_{i k}\left(\mathbf{x}_{[s]}\right) \delta^{k}, i \in[1, m]
\end{aligned}
$$

We will assume, without loss of generality, that $\operatorname{rank}_{\mathcal{K}(\delta]}\left(\mathbf{g}_{1}(\mathbf{x}, \delta)\right)=m$ (number of inputs), that is each input acts independently on the system. Starting from (14), we can thus consider $\bar{F}(\mathbf{x}, \mathbf{u}, \epsilon)=$ $\left(F(\mathbf{x})+\sum_{i=0}^{l} \sum_{j=1}^{m} G_{j i}(\mathbf{x}) u_{[0], j}(-i)\right) \epsilon(0)$. For a given $\tau(\mathbf{x}, \mathbf{u}, \delta)$, let

$$
\begin{aligned}
a d_{\bar{F}(\mathbf{x}, \mathbf{u}, 1)} \tau(\mathbf{x}, \mathbf{u}, \delta) & :=\left.a d_{\bar{F}(\mathbf{x}, \mathbf{u}, \epsilon)} \tau(\mathbf{x}, \mathbf{u}, \delta)\right|_{\epsilon(i)=1} \\
& =\dot{\tau}(\mathbf{x}, \mathbf{u}, \delta)-f(\mathbf{x}, \mathbf{u}, \delta) \tau(\mathbf{x}, \mathbf{u}, \delta)
\end{aligned}
$$

and iteratively for any $i>1$ :

$$
a d_{\bar{F}(\mathbf{x}, \mathbf{u}, 1)}^{i} \tau(\mathbf{x}, \mathbf{u}, \delta)=a d_{\bar{F}(\mathbf{x}, \mathbf{u}, 1)}^{i-1}\left(a d_{\bar{F}(\mathbf{x}, \mathbf{u}, 1)} \tau(\mathbf{x}, \mathbf{u}, \delta)\right) .
$$

Accordingly, the accessibility submodule generators introduced in [18], [19], defined (up to the sign) as,

$g_{i+1, j}\left(\mathbf{x}, \mathbf{u}^{[i-1]}, \delta\right)=\dot{g}_{i, j}\left(\mathbf{x}, \mathbf{u}^{[i-2]}, \delta\right)-f(\mathbf{x}, \mathbf{u}, \delta) g_{i, j}\left(\mathbf{x}, \mathbf{u}^{[i-2]}, \delta\right)$

are given by

$$
g_{i+1, j}\left(\mathbf{x}, \mathbf{u}^{[i-1]}, \delta\right)=a d_{\bar{F}(\mathbf{x}, \mathbf{u}, 1)}^{i} g_{1, j}(\mathbf{x}, \delta)
$$

which implies that they can be expressed in terms of Generalized Lie Brackets. In fact, particularizing to the present case Proposition 7 in the Appendix, one gets that if $s$ is the maximum delay acting on the state variable and the input variable, then setting $\bar{F}_{0}(\mathbf{x}, \delta)=\sum_{j=0}^{n s} \bar{F}_{0}^{j}(\mathbf{x}) \delta^{j}=\sum_{j=0}^{n s} F(\mathbf{x}) \delta^{j}$, for $i \leq n$, and $g_{i l}(\mathbf{x}, 0, \delta)=\sum_{p=0}^{i s} g_{i l}^{p}(\mathbf{x}, 0) \delta^{p}$, and denoting $g_{i l}^{p}(\mathbf{x}, 0)$ with $g_{i l}^{p}(0)$, then

$$
\begin{aligned}
g_{i l}(\mathbf{x}, 0, \delta) & =a d_{\bar{F}(\mathbf{x}, 0,1)}^{i-1} g_{1 l}(\mathbf{x}, \delta)=\sum_{p=0}^{i s}\left[\bar{F}_{0}^{i s}, g_{i-1, l}^{p}(0)\right]_{E_{0}} \delta^{p} \\
& =\sum_{p=0}^{i s}\left[\bar{F}_{0}^{i s}, \cdots,\left[\bar{F}_{0}^{i s}, g_{1 l}^{p}\right]_{E_{i s}}\right]_{E_{0}} \delta^{p},
\end{aligned}
$$

whereas $g_{i l}(\mathbf{x}, \mathbf{u}, \delta)=a d_{\bar{F}(\mathbf{x}, \mathbf{u}, 1)}^{i-1} g_{1 l}(\mathbf{x}, \delta)$ is given by

$$
\begin{gathered}
g_{i l}(\mathbf{x}, \mathbf{u}, \delta)=g_{i l}(\mathbf{x}, 0, \delta)+\sum_{j=1}^{m} \sum_{q=0}^{i-2} \sum_{\mu=1}^{i-1-q} \\
\sum_{k=-p-i s}^{p+i s} \sum_{\ell=0}^{i s}\left(\begin{array}{c}
i-1 \\
\mu+q
\end{array}\right) c_{\mu}^{q}\left[g_{\mu, j}^{k+\ell}(0), g_{i-\mu-q, l}^{\ell}(0)\right]_{E_{0}} \delta^{\ell} u_{j}^{(q)}(-k) \\
+m_{i}\left(\mathbf{x}, \mathbf{u}^{[i-3]}, \delta\right)
\end{gathered}
$$

where $c_{\mu}^{0}=c_{1}^{q}=1$, and for $\mu>1, q>0, c_{\mu}^{q}=c_{\mu-1}^{q}+$ $c_{\mu}^{q-1}$, and $m_{i}\left(\mathbf{x}, \mathbf{u}^{[i-3]}, \delta\right)$ is given by the linear combination, through real coefficients, of terms of the form

$\sum_{\ell}\left[g_{\mu_{1}, j_{1}}^{i_{1}+\ell}(0), \cdots,\left[g_{\mu_{\nu}, j_{\nu}}^{i_{\nu}+\ell}(0), g_{i-q, l}^{\ell}(0)\right]_{E_{i s}}\right]_{E_{0}} \delta^{\ell} \prod_{\mu=1}^{\nu} u_{j_{\mu}}^{\left(\ell_{\mu}\right)}\left(-i_{\mu}\right)$.

where $\nu \in[2, i-1], j_{\mu} \in[1, m], q=\sum_{k=1}^{\nu} \ell_{k}+\mu_{k} \leq i-1$.

Consider now the accessibility submodules $\mathcal{R}_{i}$ of $\Sigma$ introduced in [18] and defined as:

$$
\mathcal{R}_{i}\left(\mathbf{x}, \mathbf{u}^{[i-2]}, \delta\right)=\operatorname{span}_{\mathcal{K}(\delta]}\left\{\mathbf{g}_{1}(\mathbf{x}, \delta) \cdots \mathbf{g}_{i}\left(\mathbf{x}, \mathbf{u}^{[i-2]}, \delta\right)\right\} .
$$

The following result can be easily proven.

Proposition 3: If for some coefficient $\alpha(\mathbf{x}, \mathbf{u}, \delta)$, $g_{i+1, j}(\cdot) \alpha(\mathbf{x}, \mathbf{u}, \delta) \in \mathcal{R}_{i}$, then $\forall k \geq 0$ there exist coefficients $\bar{\alpha}_{k}(\mathbf{x}, \mathbf{u}, \delta)$ such that $g_{i+k+1, j}(\cdot) \bar{\alpha}_{k}(\mathbf{x}, \mathbf{u}, \delta) \in \mathcal{R}_{i}$.

A direct consequence is the following. 
Proposition 4: Let $k=\operatorname{rank}_{\mathcal{K}(\delta]}\left(\mathcal{R}_{n}(\mathbf{x}, \mathbf{u}, \delta)\right)$ for some $\mathbf{u}$ and set $\mathcal{G}(\delta]=\operatorname{span}_{\mathcal{K}(\delta]}\left\{\mathbf{g}_{1}(\mathbf{x}, \delta), \cdots, \mathbf{g}_{n}(\mathbf{x}, 0, \delta)\right\}$. Then $\overline{\mathcal{G}}(\delta]$, the involutive closure of $\mathcal{G}(\delta]$ has dimension $k$.

Proof. By construction, due to the expression of the $g_{i, l}(\mathbf{x}, \mathbf{u}, \delta)$ 's, we have that $g_{i, l}(\mathbf{x}, \mathbf{u}, \delta) \in \overline{\mathcal{G}}(\delta]$.

Let $\tau(\mathbf{x}, \delta)$, be such that for some $\alpha(\mathbf{x}, \mathbf{u}, \delta) \neq 0$, $\tau(\mathbf{x}, \delta) \alpha(\cdot) \in \mathcal{R}_{n}(\mathbf{x}, \mathbf{u}, \delta)$. Then, for some $\bar{\alpha}^{i}(\mathbf{x}, \mathbf{u}, \delta) \neq 0$, also

$$
\left(a d_{\bar{F}(\mathbf{x}, \mathbf{u}, 1)}^{i} \tau\right) \bar{\alpha}^{i}(\mathbf{x}, \mathbf{u}, \delta) \in \mathcal{R}_{n}(\mathbf{x}, \mathbf{u}, \delta)
$$

In fact, since $\tau(\mathbf{x}, \delta) \alpha=\sum_{\nu=1}^{m} \sum_{j=1}^{k} g_{j \nu}(\mathbf{x}, \mathbf{u}, \delta) \beta_{j \nu}$, from (34) in the Appendix,

$$
\begin{aligned}
a d_{\bar{F}(\mathbf{x}, \mathbf{u}, 1)}^{i}(\tau \alpha) & =\sum_{\ell=0}^{i}\left(\begin{array}{l}
i \\
\ell
\end{array}\right) a d_{\bar{F}(\mathbf{x}, \mathbf{u}, 1)}^{i-\ell} \tau(\mathbf{x}, \delta) \alpha^{(\ell)} \\
& =\sum_{\nu=1}^{m} \sum_{j=0}^{k+i} g_{j, \nu}(\mathbf{x}, \mathbf{u}, \delta) \bar{\beta}_{j, \nu}(\mathbf{x}, \mathbf{u}, \delta)
\end{aligned}
$$

which iteratively proves (22) for any $i>0$. Consider now $\tau_{1}(\mathbf{x}, \delta)$, such that for some $\alpha_{1} \neq 0, \tau_{1}\left(\mathbf{x}_{[s]}, \delta\right) \alpha_{1} \in$ $\mathcal{R}_{n}(\mathbf{x}, \mathbf{u}, \delta)$. From (35),

$$
\begin{gathered}
a d_{\bar{F}(\mathbf{x}, \mathbf{u}, 1)}^{k+1} \tau_{1}(\mathbf{x}, \delta)=a d_{\bar{F}(\mathbf{x}, 0,1)}^{k+1} \tau_{1}(\mathbf{x}, \delta)+\sum_{j=1}^{m} \sum_{q=0}^{k-1} \sum_{\ell, p=0}^{(k+1) s} \\
\sum_{\mu=1}^{k-q}\left(\begin{array}{c}
k \\
\mu+q
\end{array}\right) c_{\mu}^{q}\left[g_{\mu, j}^{\ell+p}(0), \tau_{k+1-\mu-q}^{\ell}\right]_{E_{0}} \delta^{\ell} u_{j,[0]}^{(q)}(-p) \\
+m_{k+1}\left(\mathbf{x}, \mathbf{u}^{[k-2]}, \delta\right)
\end{gathered}
$$

According to the previous discussion, since $\tau_{1} \alpha_{1} \in \mathcal{R}_{n}$ then there exists $\bar{\alpha}^{k+1} \neq 0$, such that $a d_{\bar{F}(\mathbf{x}, \mathbf{u}, 1)}^{k+1} \tau_{1}(\mathbf{x}, \delta) \bar{\alpha}^{k+1}=$ $\sum_{\nu=1}^{m} \sum_{j=1}^{k} g_{j, \nu}(\mathbf{x}, \mathbf{u}, \delta) \alpha_{j, \nu}$. It follows that, since for $i \leq k$ $g_{i, \nu}(\mathbf{x}, \mathbf{u}, \delta)$ does not depend on $u_{j,[0]}^{(k-1)}(-p)$, for any $p$

$$
\begin{aligned}
& \frac{\partial}{\partial u_{j,[0]}^{(k-1)}(-p)}\left(a d_{\bar{F}(\mathbf{x}, \mathbf{u}, 1)}^{k+1} \tau_{1}(\mathbf{x}, \delta) \bar{\alpha}^{k+1}\right)= \\
& \frac{\partial a d_{\bar{F}(\mathbf{x}, \mathbf{u}, 1)}^{k+1} \tau_{1}(\mathbf{x}, \delta)}{\partial u_{j,[0]}^{(k-1)}(-p)} \bar{\alpha}^{k+1}+a d_{\bar{F}(\mathbf{x}, \mathbf{u}, 1)}^{k+1} \tau_{1}(\mathbf{x}, \delta) \frac{\partial \bar{\alpha}^{k+1}}{\partial u_{j,[0]}^{(k-1)}(-p)} \\
& =\sum_{\nu=1}^{m} \sum_{i=1}^{k} g_{i, \nu}(\mathbf{x}, \mathbf{u}, \delta) \frac{\partial \alpha_{i \nu}}{\partial u_{j,[0]}^{(k-1)}(-p)}
\end{aligned}
$$

that is, using the expression of $a d_{\bar{F}(\mathbf{x}, \mathbf{u}, 1)}^{k+1} \tau_{1}(\mathbf{x}, \delta)$, given by (23), and setting $\hat{\alpha}_{j p}^{k+1}=\frac{\partial \bar{\alpha}^{k+1}}{\partial u_{j,[0]}^{(k-1)}(-p)}$

$$
\begin{gathered}
\sum_{\ell=0}^{(k+1) s} c_{1}^{k-1}\left[g_{\mu, j}^{\ell+p}(0), \tau_{1}^{\ell}\right]_{E_{0}} \delta^{\ell} \bar{\alpha}^{k+1}+a d_{\bar{F}(\mathbf{x}, \mathbf{u}, 1)}^{k+1} \tau_{1}(\mathbf{x}, \delta) \hat{\alpha}_{j p}^{k+1} \\
=\sum_{\nu=1}^{m} \sum_{i=1}^{k} g_{i, \nu}(\mathbf{x}, \mathbf{u}, \delta) \frac{\partial \alpha_{i, \nu}}{\partial u_{j,[0]}^{(k-1)}(-p)}
\end{gathered}
$$

which shows that for an appropriate $\beta \neq 0$,

$$
\sum_{\ell=0}^{(k+1) s}\left[g_{\mu, j}^{\ell+p}(0), \tau_{1}^{\ell}\right]_{E_{0}} \delta^{\ell} \beta=\sum_{\nu=1}^{m} \sum_{i=1}^{k} g_{i \nu}(\mathbf{x}, \mathbf{u}, \delta) \bar{\alpha}_{i \nu}
$$

whenever $\tau_{1}(\mathbf{x}, \delta)$ satisfies for some $\alpha_{1} \neq 0, \tau_{1}(\mathbf{x}, \delta) \alpha_{1} \in \mathcal{R}_{n}$.

Set $\tau_{1}(\mathbf{x}, \delta)=g_{1 i}(\mathbf{x}, \delta)$. Equation (24) implies that for all $i, j \in[1, m]$, and for all $\ell \in[0,(k+1) s]$ and for some $\beta \neq$ $0, \sum_{\ell}\left[g_{1 j}^{\ell+p}, g_{1 i}^{\ell}\right]_{E_{0}} \delta^{\ell} \beta \in \mathcal{R}_{n}$. As a consequence, due to the structure of $g_{2 i}(\mathbf{x}, \mathbf{u}, \delta)$, also $g_{20, i}(\mathbf{x}, \delta) \beta \in \mathcal{R}_{n}$. Iteratively one gets that each element of $\overline{\mathcal{G}}(\delta]$, the involutive closure of $\mathcal{G}(\delta]$, post multiplied by an appropriate non zero coefficient is in $\mathcal{R}_{n}$. As a consequence, $\overline{\mathcal{G}}(\delta]$ has rank $k . \triangleleft$

Let us now recall that a function $\lambda\left(\mathbf{x}_{[\bar{s}]}\right)$ has finite relative degree $\mathrm{k}$ if $\forall l \in[1, m]$, and $\forall i \in[1, k-1]$

$$
L_{g_{i l}^{j}\left(\mathbf{x}, \mathbf{u}^{[i-2]}\right)} \lambda\left(\mathbf{x}_{[\bar{s}]}\right)=0, \forall j \in\left[0, \bar{s}+\beta_{i}\right], \forall \mathbf{u}^{[i-2]},
$$

and there exists an index $l \in[1, m]$ such that

$$
L_{g_{k l}^{j}\left(\mathbf{x}, \mathbf{u}^{[k-2]}\right)} \lambda\left(\mathbf{x}_{[\bar{s}]}\right) \neq 0 \text { for some } j \in\left[0, \bar{s}+\beta_{k}\right] .
$$

It immediately follows that a function $\lambda(\mathbf{x})$ has relative degree $k>0$ if

$$
\begin{aligned}
& d \lambda(\mathbf{x}) \perp \mathcal{R}_{k-1}\left(\mathbf{x}, \mathbf{u}^{[k-3]}, \delta\right) \\
& d \lambda(\mathbf{x}) g_{k, \ell}\left(\mathbf{x}, \mathbf{u}^{[k-2]}, \delta\right) \neq 0 \text { for some } \ell \in[1, m] .
\end{aligned}
$$

The following results gives conditions, which are independent of the control $u$, for a function to have relative degree $k$.

Proposition 5: A function $\lambda(\mathbf{x})$ has relative degree $k>0$ if and only if $\forall l \in[1, m]$,

$$
d \lambda(\mathbf{x}) g_{i l}(\mathbf{x}, 0, \delta)=0, \forall i \leq k-1,
$$

and for some $l \in[1, m]$,

$$
d \lambda(\mathbf{x}) g_{k l}(\mathbf{x}, 0, \delta) \neq 0 .
$$

Proof. The proof is immediate if one refers to the expression of $g_{i l}(\mathbf{x}, \mathbf{u}, \delta)$ given by (20). In fact if the function $\lambda(\mathbf{x})$ has relative degree $k$, then equation (25) must be satisfied for $i \in[1, k-1]$. In particular it must be satisfied for $u=0$, which leads the necessity of (28). Consequently one also gets that setting $\tau=$ $\left[g_{i-\mu-\ell, l}^{p}(0)\left[g_{\mu_{1}, j_{1}}^{i_{1}}(0) \cdots\left[g_{\mu_{\nu-1}, j_{\nu-1}}^{i_{\nu-1}}(0), g_{\mu_{\nu}, j_{\nu}}^{i_{\nu}}(0)\right]_{E_{n s}} \cdots\right]_{E_{n s}}\right.$ with $\mu=\sum_{i} \mu_{i}, \ell \in[0, i-\mu-1]$, then $d \lambda(\mathbf{x}) \tau=0$, which proves, due to (20), that equation (26) is satisfied only if (29) is satisfied.

Conversely, assume that equation (28) and (29) are satisfied, then, due to (28) and (20), one gets immediately that (25) is satisfied for $i \in[1, k-1]$, whereas (29) implies that necessarily (26) must be satisfied, so that the function $\lambda(\mathbf{x})$ has relative degree $k . \triangleleft$

It follows that any non constant autonomous function $\lambda\left(\mathbf{x}_{[\bar{s}]}\right) \in \mathcal{K}$ has infinite relative degree, so that the following result is of interest.

Lemma 1: Given the dynamics (14), the relative degree of a non constant function $\lambda\left(\mathbf{x}_{[\bar{s}]}\right) \in \mathcal{K}$ is greater than $n$ if and only if it is infinite. 
Proof. Of course if the relative degree is infinite it is greater than $n$. The converse follows immediately by noting that by assumption $\lambda\left(\mathbf{x}_{[\bar{s}]}\right) \perp \mathcal{R}_{n}\left(\mathbf{x}, \mathbf{u}^{[n-2]}, \delta\right)$ and that $\forall \beta \geq 1$, there exists a coefficient $\alpha_{\beta}$ such that $g_{n+\beta, j}\left(\mathbf{x}, \mathbf{u}^{[n+\beta-2]}, \delta\right) \alpha_{\beta} \in$ $\mathcal{R}_{n}\left(\mathbf{x}, \mathbf{u}^{[n-2]}, \delta\right), \forall j \in[1, m] . \triangleleft$

The following result gives a criterion to test the accessibility of a given system.

Theorem 5: The dynamics (14) is locally accessible if and only if the following equivalent statements hold true:

- $\mathcal{R}_{n}\left(\mathbf{x}, \mathbf{u}^{[n-2]}, \delta\right)$ is torsion free over $\mathcal{K}(\delta]$,

- $\operatorname{rank}_{\mathcal{K}(\delta]} \mathcal{R}_{n}\left(\mathbf{x}, \mathbf{u}^{[n-2]}, \delta\right)=n$ for some $\mathbf{u}^{[n-2]}$,

- $\operatorname{dim} \overline{\mathcal{G}}(\delta)=n$.

Proof. Of course if $\mathcal{R}_{n}\left(\mathbf{x}, \mathbf{u}^{[n-2]}, \delta\right)$ is torsion free over $\mathcal{K}(\delta]$, then there is no nonzero element which annihilates $\mathcal{R}_{n}\left(\mathbf{x}, \mathbf{u}^{[n-2]}, \delta\right)$, that is $\operatorname{rank}_{\mathcal{K}(\delta]} \mathcal{R}_{n}\left(\mathbf{x}, \mathbf{u}^{[n-2]}, \delta\right)=n$. Consequently, there cannot exist any function with infinite relative degree, $\operatorname{dim} \overline{\mathcal{G}}(\delta)=n$ and the given system is accessible. As for the converse, assume that $\mathcal{R}_{n}\left(\mathbf{x}, \mathbf{u}^{[n-2]}, \delta\right)$ is not torsion free over $\mathcal{K}(\delta]$. Then $\operatorname{rank}_{\mathcal{K}(\delta]} \mathcal{R}_{n}\left(\mathbf{x}, \mathbf{u}^{[n-2]}, \delta\right)=$ $k<n$ for all possible choices of $\mathbf{u}^{[k-2]}$. According to Proposition $4, \overline{\mathcal{G}}(\delta]$ the involutive closure of $\mathcal{G}(\delta]=$ $\operatorname{span}_{\mathcal{K}(\delta]}\left\{\mathbf{g}_{1}(\mathbf{x}, \delta), \mathbf{g}_{2}(\mathbf{x}, 0, \delta), \cdots, \mathbf{g}_{n}(\mathbf{x}, 0, \delta)\right\}$ has rank $k$, so that there exist $n-k$ exact differentials in the left annihilator, independent over $\mathcal{K}(\delta]$. Due to Proposition 5 the corresponding functions have infinite relative degree. $\triangleleft$

\section{A. A Canonical decomposition with respect to accessibility}

Theorem 5, gives a criterion to test accessibility of a given system. If $\operatorname{rank}_{\mathcal{K}(\delta]} \mathcal{R}_{n}(\mathbf{x}, \mathbf{u}, \delta)<n$ the system is not accessible and there exist $n-k$ independent functions $\varphi_{1}(\mathbf{x}), \cdots, \varphi_{n-k}(\mathbf{x})$ which are characterized by an infinite relative degree.

We are thus interested in characterizing the non accessible part of the system, that is defining a bicausal change of coordinates which decomposes in the new coordinates the given system into two parts, one of which represents the non accessible subsystem.

Consider $\mathcal{G}(\delta]=\operatorname{span}_{\mathcal{K}(\delta]}\left\{\mathbf{g}_{1}(\mathbf{x}, \delta), \cdots, \mathbf{g}_{n}(\mathbf{x}, 0, \delta)\right\}$ and since the elements of the submodule are by construction causal, consider for $i \geq 0$, the sequence of distributions $\mathcal{G}_{i}:=\mathcal{G}_{[0, i+s]} \subset \operatorname{span}\left\{\frac{\partial}{\partial \mathbf{x}_{[0]}}, \cdots, \frac{\partial}{\partial \mathbf{x}_{[0]}(-i-s)}\right\}$ defined $\mathcal{G}_{i}=\operatorname{span}\left(\begin{array}{cccccc}\mathbf{g}^{0}\left(\mathbf{x}_{[s]}\right) & \cdots & \mathbf{g}^{\ell}\left(\mathbf{x}_{[s]}\right) & 0 & & 0 \\ 0 & \ddots & & \ddots & & \\ \vdots & 0 & \mathbf{g}^{0}\left(\mathbf{x}_{[s]}(-i)\right) & \cdots & \mathbf{g}^{\ell}\left(\mathbf{x}_{[s]}(-i)\right) & 0 \\ 0 & \cdots & 0 & & & I_{n s}\end{array}\right)$,

where $\ell$ represents the maximum degree in $\delta$ and $s$ the maximum delay in $x$ which are present in the $g_{i, j}$ 's. $\mathcal{G}_{i}$ is a distribution in $\mathbb{R}^{n(s+i+1)}$ as well as its involutive closure $\overline{\mathcal{G}_{i}}$. Let $\rho_{i}=\operatorname{rank}\left(\overline{\mathcal{G}}_{i}\right)$, with $\rho_{-1}=n s$. The following result can be stated.
Proposition 6: Assume that the system $\Sigma$, given by (14), is not accessible, i.e., $\operatorname{rank} \mathcal{R}_{n}(\mathbf{x}, \mathbf{u}, \delta)=k<n$, then the following facts hold true:

i) The system $\Sigma$ possesses $n-k$ independent (over $\mathcal{K}(\delta]$ ) autonomous exact differentials.

ii) A canonical basis for $\overline{\mathcal{G}}_{i}^{\perp}$ is defined for $i \geq 0$ as follows. Let $d \lambda_{0}\left(\mathbf{x}_{[0]}\right)$ be such that $\operatorname{span}\left\{d \lambda_{0}\left(\mathbf{x}_{[0]}\right)\right\}=\overline{\mathcal{G}}_{0}^{\perp}$, with $\operatorname{rank}\left(d \lambda_{0}\right)=\mu_{0}=\rho_{0}-\rho_{-1}$.

Let $d \lambda_{1}\left(\mathbf{x}_{[1]}\right) \quad \notin \quad \overline{\mathcal{G}}_{0}^{\perp}$, with $\operatorname{rank}\left(d \lambda_{1}\right)=$ $\mu_{1}=\rho_{1}-2 \rho_{0}+\rho_{-1}$, be such that $\operatorname{span}\left\{d \lambda_{0}\left(\mathbf{x}_{[0]}\right), d \lambda_{0}\left(\mathbf{x}_{[0]}(-1)\right), d \lambda_{1}\left(\mathbf{x}_{[1]}\right)\right\}=\overline{\mathcal{G}}_{1}^{\perp}$.

More generally, let $d \lambda_{i}\left(\mathbf{x}_{[i]}\right) \notin \overline{\mathcal{G}}_{i-1}^{\perp}$, with $\operatorname{rank}\left(d \lambda_{i}\right)=\mu_{i}=\rho_{i}-2 \rho_{i-1}+\rho_{i-2}$ be such that $\operatorname{span}\left\{d \lambda_{\mu}\left(\mathbf{x}_{[\mu]}(-j)\right), \mu \in[0, i], j \in[0, i-\mu]\right\}=\overline{\mathcal{G}}_{i}^{\perp}$.

iii) Let $\bar{\ell}$ represent the maximum degree in $\delta$ and $\bar{s}$ the maximum delay in $x$ in $\mathcal{R}_{n}(\mathbf{x}, \mathbf{u}, \delta)$. Then $\exists \gamma \leq \bar{s}+k \bar{\ell}$ such that any other autonomous function $\lambda(\mathbf{x})$ satisfies

$$
d \lambda(\mathbf{x}) \in \operatorname{span}_{\mathcal{K}(\delta]}\left\{d \lambda_{0}(\mathbf{x}), \cdots, d \lambda_{\gamma}(\mathbf{x})\right\}
$$

that is $\overline{\mathcal{G}}_{\gamma}$ characterizes completely all the independent autonomous functions of $\Sigma$.

Proof. i) is a direct consequence of Proposition 4. ii) is a direct consequence of Lemma 2 in the Appendix, where $\Delta_{i}=\mathcal{G}_{i}$ is causal by assumption, thus ensuring that the left annihilator is causal also. Finally, iii) is a direct consequence of Lemma 4 in the Appendix. $\triangleleft$

Theorem 6: Consider the continuous-time system (14). Let $\gamma$ be the smallest index such that any autonomous function $\lambda(\mathbf{x})$ associated to the given system, satisfies $d \lambda(\mathbf{x}) \in$ $\operatorname{span}_{\mathcal{K}(\delta]}\left\{d \lambda_{0}\left(\mathbf{x}_{[0]}\right), \cdots, d \lambda_{\gamma}\left(\mathbf{x}_{[\gamma]}\right)\right\}$ where

$$
\begin{gathered}
\operatorname{span}\left\{d \lambda_{0}(\mathbf{x})\right\}=\overline{\mathcal{G}}_{0}^{\perp} \\
\operatorname{span}\left\{d \lambda_{0}, d \lambda_{0}(\mathbf{x}(-1)), d \lambda_{1}(\mathbf{x})\right\}=\overline{\mathcal{G}}_{1}^{\perp}, \quad d \lambda_{1}\left(\mathbf{x}_{[1]}\right) \notin \overline{\mathcal{G}}_{0}^{\perp} \\
\vdots \\
\operatorname{span}\left\{d \lambda_{i}(\mathbf{x}(-j)), i \in[0, \gamma], j \in[0, \gamma-i]\right\}=\overline{\mathcal{G}}_{\gamma}^{\perp}, \\
d \lambda_{\gamma}\left(\mathbf{x}_{[\gamma]}\right) \notin \overline{\mathcal{G}}_{\gamma-1}^{\perp}
\end{gathered}
$$

then

$$
\begin{aligned}
& \text { 1.) } \exists d \lambda_{\gamma+1}(\mathbf{x}) \text { such that } \\
& d \mathbf{z}_{[0]}=\left(\begin{array}{c}
d z_{1,[0]} \\
\vdots \\
d z_{\gamma+1,[0]} \\
d z_{\gamma+2,[0]}
\end{array}\right)=\left(\begin{array}{c}
d \lambda_{0}\left(\mathbf{x}_{[0]}\right) \\
\vdots \\
d \lambda_{\gamma}\left(\mathbf{x}_{[\gamma]}\right) \\
d \lambda_{\gamma+1}(\mathbf{x})
\end{array}\right)=T(\mathbf{x}, \delta) d \mathbf{x}_{[0]}
\end{aligned}
$$

defines a bicausal change of coordinates.

2.) In the above defined coordinates $\mathbf{z}_{[0]}=\phi(\mathbf{x})$ such that 


$$
\begin{gathered}
d \mathbf{z}_{[0]}=T(\mathbf{x}, \delta) d \boldsymbol{x}_{[0]} \text { the system reads } \\
\dot{z}_{1,[0]}=f_{1}\left(z_{1,[\bar{s}]}, \cdots, z_{\gamma,[\bar{s}]}\right) \\
\vdots \\
\dot{z}_{\gamma+1,[0]}=f_{\gamma+1}\left(z_{1,[\bar{s}]}, \cdots, z_{\gamma,[\bar{s}]}\right) \\
\dot{z}_{\gamma+2,[0]}=f_{\gamma+2}(\mathbf{z})+\sum_{i=0}^{\bar{s}} \sum_{j=1}^{m} \tilde{G}_{j i}(\mathbf{z}) u_{j,[0]}(-i)
\end{gathered}
$$

Moreover the dynamics associated to $\left(z_{1}, \cdots z_{\gamma+1}\right)^{T}$ represents the largest non accessible dynamics.

Proof. By construction $\operatorname{span}_{\mathcal{K}(\delta]}\left\{d \lambda_{0}(\mathbf{x}), \cdots, d \lambda_{\gamma}(\mathbf{x})\right\}$ is closed and its right annihilator is causal so that, according to Theorem 2, it is possible to compute $\lambda_{\gamma+1}(\mathbf{x})$ such that

$$
\mathbf{z}_{[0]}=\left(\begin{array}{c}
d z_{1,[0]} \\
\vdots \\
d z_{\gamma+1,[0]} \\
d z_{\gamma+2,[0]}
\end{array}\right)=\left(\begin{array}{c}
d \lambda_{0}\left(\mathbf{x}_{[0]}\right) \\
\vdots \\
d \lambda_{\gamma}\left(\mathbf{x}_{[\gamma]}\right) \\
d \lambda_{\gamma+1}(\mathbf{x})
\end{array}\right)=T(\mathbf{x}, \delta) d \mathbf{x}_{[0]}
$$

is a bicausal change of coordinates.

Consider $\dot{\lambda}_{i}(\mathbf{x})$ for $i \in[0, \gamma]$. By construction,

$$
d \lambda_{i}(\mathbf{x}) g_{1, j}(\mathbf{x}, \delta)=0, \quad i \in[0, \gamma] .
$$

Consequently if $\alpha$ is the maximum delay in $\lambda_{i}(\mathbf{x})$, that is $\lambda_{i}:=\lambda_{i}\left(\mathbf{x}_{[\alpha]}\right)$, then

$$
\dot{\lambda}_{i}\left(\mathbf{x}_{[\alpha]}\right)=\sum_{j=0}^{\alpha} \frac{\partial \lambda_{i}\left(\mathbf{x}_{[\alpha]}\right)}{\partial \mathbf{x}_{[0]}(-j)} F(\mathbf{x}(-j)), \quad i \in[0, \gamma] .
$$

Let $d \lambda_{i}(\mathbf{x})=\Lambda_{i}(\mathbf{x}, \delta) d \mathbf{x}_{[0]}$, then

$$
\begin{aligned}
& d \dot{\lambda}_{i}(\mathbf{x})=\dot{\Lambda}_{i}(\mathbf{x}, \delta) d \mathbf{x}_{[0]}+\Lambda_{i}(\mathbf{x}, \delta) d \dot{\mathbf{x}}_{[0]} \\
& =\dot{\Lambda}_{i}(\mathbf{x}, \delta) d \mathbf{x}_{[0]}+\Lambda_{i}(\mathbf{x}, \delta) f(\mathbf{x}, \mathbf{u}, \delta) d \mathbf{x}_{[0]}=\Gamma(\mathbf{x}, \delta) d \mathbf{x}_{[0]}
\end{aligned}
$$

By assumption, for any $k \geq 1$ and any $j \in[1, m]$,

$$
\Lambda_{i}(\mathbf{x}, \delta) g_{k, j}(\mathbf{x}, \mathbf{u}, \delta)=0
$$

so that derivating both sides, one gets $\forall k \geq 1, j \in[1, m]$,

$$
\begin{gathered}
0=\dot{\Lambda}_{i}(\mathbf{x}, \delta) g_{k, j}(\mathbf{x}, \mathbf{u}, \delta)+\Lambda_{i}(\mathbf{x}, \delta) \dot{g}_{k, j}(\mathbf{x}, \mathbf{u}, \delta)= \\
\dot{\Lambda}_{i}(\mathbf{x}, \delta) g_{k, j}(\mathbf{x}, \mathbf{u}, \delta)+\Lambda_{i}(\mathbf{x}, \delta) f(\mathbf{x}, \mathbf{u}, \delta) g_{k, j}(\mathbf{x}, \mathbf{u}, \delta)
\end{gathered}
$$

It follows that for any $k \geq 1$ and any $j \in[1, m]$, by considering that $d \dot{\lambda}_{i}(\mathbf{x})$ is given by (32), then, due to (33),

$$
\begin{gathered}
\Gamma(\mathbf{x}, \delta) g_{k, j}(\mathbf{x}, \mathbf{u}, \delta)= \\
\dot{\Lambda}_{i}(\mathbf{x}, \delta) g_{k, j}(\mathbf{x}, \mathbf{u}, \delta)+\Lambda_{i}(\mathbf{x}, \delta) f(\mathbf{x}, \mathbf{u}, \delta) g_{k, j}(\mathbf{x}, \mathbf{u}, \delta)=0
\end{gathered}
$$

As a consequence $d \dot{\lambda}_{i} \in \operatorname{span}_{\mathcal{K}(\delta]}\left\{d \lambda_{0}\left(\mathbf{x}_{[0]}\right), \cdots, d \lambda_{\gamma}\left(\mathbf{x}_{[\gamma]}\right)\right\}$ for any $i \in[0, \gamma]$. Accordingly in the coordinates (31) the system necessarily reads (30).

$\triangleleft$

\section{EXAMPLES}

\section{The JAK-STAT signaling pathway Model}

In Biology, the JAnus Kinase-Signal Transducer and Activator of Transcription (JAK-STAT) signaling pathway transmits information from outside a cell, through the cell membrane, to cause DNA transcription in the cell. The dynamic model of the JAK-STAT given in [25] is considered hereafter.

A kinase is a type of enzyme which enables phosphorylation, i.e. the transfer of phosphate groups a specific substrate (here STAT-5). In the state model below, $x_{1}$ stands for the unphosphorylated monomeric STAT- 5 and $x_{2}$ for the phosphorylated monomeric STAT-5. This transfer occurs under the control action $u$ which denotes the amount of activated Epo-receptors. In addition, $x_{3}$ represents the phosphorylated dimeric STAT-5 in the cytoplasm while $x_{4}$ is the phosphorylated dimeric STAT-5 in the nucleus. All together, the STAT-5 cycling model can be described as follows:

$$
\begin{aligned}
& \dot{x}_{1,[0]}=-k_{1} x_{1,[0]} u+2 k_{4} x_{3,[0]}(-1) \\
& \dot{x}_{2,[0]}=k_{1} x_{1,[0]} u-k_{2} x_{2,[0]}^{2}+2 k_{3}^{\prime} x_{3,[0]} \\
& \dot{x}_{3,[0]}=-k_{3} x_{3,[0]}+k_{2} x_{2,[0]}^{2} / 2-k_{3}^{\prime} x_{3,[0]} \\
& \dot{x}_{4,[0]}=k_{3} x_{3,[0]}-k_{4} x_{3,[0]}(-1)
\end{aligned}
$$

The differential representation of the model

$$
d \dot{\mathbf{x}}_{[0]}=f(\mathbf{x}, \mathbf{u}, \delta) d \mathbf{x}_{[0]}+g_{1}(\mathbf{x}, \delta) d \mathbf{u}_{[0]}
$$

is characterized by

$$
\begin{aligned}
f(\mathbf{x}, \mathbf{u}, \delta) & =\left(\begin{array}{cccc}
-k_{1} u_{[0]} & 0 & 2 k_{4} \delta & 0 \\
k_{1} u_{[0]} & -2 k_{2} x_{2,[0]} & 2 k_{3}^{\prime} & 0 \\
0 & k_{2} x_{2,[0]} & -k_{3}-k_{3}^{\prime} & 0 \\
0 & 0 & k_{3}-k_{4} \delta & 0
\end{array}\right) \\
g_{1}(\mathbf{x}, \delta) & =\left(\begin{array}{c}
-k_{1} x_{1,[0]} \\
k_{1} x_{1,[0]} \\
0 \\
0
\end{array}\right)
\end{aligned}
$$

Consequently, denoting for simplicity $x_{i,[0]}$ by $x_{i}$, then

$$
\begin{gathered}
g_{2}(\mathbf{x}, \mathbf{u}, \delta)=g_{1}(\mathbf{x}, \delta) 2 k_{4} \frac{x_{3}(-1)}{x_{1}}-\left(\begin{array}{c}
0 \\
-2 \\
1 \\
0
\end{array}\right) k_{1} k_{2} x_{1} x_{2} \\
g_{3}(\mathbf{x}, \mathbf{u}, \delta)=-g_{2}(\mathbf{x}, \mathbf{u}, \delta)\left(\frac{\dot{x}_{1} x_{2}+x_{1} \dot{x}_{2}}{x_{1} x_{2}}-2 k_{4} \frac{x_{3}(-1)}{x_{1}}\right) \\
2 k_{4} \delta \\
+g_{1}(\mathbf{x}, \delta) \alpha_{0}+\left(\begin{array}{c}
4 k_{2} x_{2}+2 k_{3}^{\prime} \\
-2 k_{2} x_{2}-k_{3}-k_{3}^{\prime} \\
k_{3}-k_{4} \delta
\end{array}\right) k_{1} k_{2} x_{1} x_{2} \\
g_{4}(\mathbf{x}, \mathbf{u}, \delta)=\sum_{i=1}^{3} g_{i}\left(\mathbf{x}, \mathbf{u}^{[i-2]}, \delta\right) \alpha_{i}(\mathbf{x}, \mathbf{u}, \delta)
\end{gathered}
$$

where $\quad \alpha_{0}=k_{4}\left(2 \frac{\dot{x}_{3}(-1)}{x_{1}}-\frac{x_{3}(-1)}{x_{1}^{2}} \dot{x}_{1}+x_{3}(-1) \frac{\dot{x}_{2}}{x_{1} x_{2}}\right)$ whereas the $\alpha_{i}$ 's, $i \in[1,3]$ are appropriate coefficients. Since $\operatorname{rank}\left(\mathcal{R}_{n}\right)=3$, the system is not completely accessible. One gets that $\lambda\left(\mathbf{x}_{[0]}\right)=x_{1}+x_{2}+2 x_{3}+2 x_{4}$. Of course any 
linear delay-free basis completion will satisfy the bicausality condition. So we can take

$$
z_{[0]}=\left(\begin{array}{cccc}
1 & 0 & 0 & 0 \\
0 & 1 & 0 & 0 \\
0 & 0 & 1 & 0 \\
1 & 1 & 2 & 2
\end{array}\right) x_{[0]}
$$

In these new coordinates the system reads

$$
\begin{aligned}
& \dot{z}_{1,[0]}=-k_{1} z_{1,[0]} u_{[0]}+2 k_{4} z_{3,[0]}(-1) \\
& \dot{z}_{2,[0]}=k_{1} z_{1,[0]} u_{[0]}-k_{2} z_{2,[0]}^{2}+2 k_{3}^{\prime} z_{3,[0]} \\
& \dot{z}_{3,[0]}=-k_{3} z_{3,[0]}+k_{2} z_{2,[0]}^{2} / 2-k_{3}^{\prime} z_{3,[0]} \\
& \dot{z}_{4,[0]}=0
\end{aligned}
$$

\section{The Chained Form Model}

Example 4: Consider the two dimensional system

$$
\dot{\mathbf{x}}_{[0]}=g\left(\mathbf{x}_{[1]}\right) u_{[0]}=\left(\begin{array}{c}
x_{2,[0]}(-1) \\
1
\end{array}\right) u_{[0]},
$$

where a delay is introduced on $x_{2}$. It is easily verified that the presence of the delay renders the system fully accessible, as opposite to the delay-free case ([1], [21], [24]).

In fact, through straightforward computations one has that

$g_{1}(\mathbf{x}, \delta)=\left(\begin{array}{c}x_{2,[0]}(-1) \\ 1\end{array}\right), g_{2}(\mathbf{x}, \mathbf{u}, \delta)=\left(\begin{array}{c}u_{[0]}(-1)-u_{[0]} \delta \\ 0\end{array}\right)$,

which shows that $\mathcal{R}_{n}$ has full rank for $u_{[0]}(-1)$ and $u_{[0]}$ different from zero. An extensive discussion on this topic can be found in [4], [17].

\section{Conclusions}

A full characterization of accessibility was derived for nonlinear time-delay systems. In addition, it has been shown that it is always possible to decompose any system within this class into an autonomous or non accessible subsystem and an accessible one. Such a decomposition is not always possible with respect to observability. One mathematical key tool is provided by the basis completion result. The so-called geometric approach is successfully extended and adapted for this class of nonlinear time-delay systems. Technical results on integrability are interesting by their own as they impact numerous potential future results in the theory of nonlinear time-delay control systems.

\section{REFERENCES}

[1] M. Bloch. Nonholonomic Mechanics and Control, Springer-Verlag, New York, 2003.

[2] C. Califano, L. A. Márquez-Martínez, C. H. Moog. Extended Lie Brackets for Nonlinear Time-Delay Systems, IEEE Trans. Automat. Control, vol. 56, pp. 2213-2218, 2011.

[3] C. Califano, L.A. Marquez-Martinez and C.H. Moog, On the observer canonical form for time-delay systems, Proc. of 18th IFAC World Congress, Milan, Italy, vol. 18, pp.3855-3860, 2011.

[4] C. Califano, S. Li, C.H. Moog, Controllability of driftless nonlinear time-delay systems Syst. and Contr. Lett., vol. 62, pp. 294-301, 2013.

[5] C. Califano, C.H. Moog, Coordinates transformations in nonlinear timedelay systems , Proc. of 53rd IEEE CDC, Los Angeles, USA, pp.475480, 2014.
[6] G. Conte, A.M. Perdon. The disturbance decoupling problem for systems over a ring, SIAM, J. Contr. Optimiz., vol. 33, pp. 750-764, 1995.

[7] M. Fliess, H. Mounier. Controllability and observability of linear delay systems: An algebraic approach, ESAIM COCV, vol. 3, pp. 301-314, 1998.

[8] A. Germani, C. Manes and P. Pepe, Linearization of input-output mapping for nonlinear delay systems via static state feedback, CESA '96 IMACS Multiconference, Lille, France, vol. 1, pp. 599-602, 1996.

[9] K. Gu, V.L. Kharitonov, J. Chen, Stability of Time-Delay Systems, Birkhaüser, Boston, 2003.

[10] R. Hermann. On the Accessibility Problem in Control Theory, in J. P. Lasalle \& S. L. Efschetz, eds., International Symposium on Nonlinear Differential Equations and Nonlinear Mechanics, Academic Press, pp. 325-332, 1963.

[11] A. Isidori, Nonlinear Control Systems, Springer Verlag, $3^{r d}$ Ed., 1995.

[12] S. Islam, X.P. Liu, A. El Saddik, Teleoperation Systems With Symmetric and Unsymmetric Time Varying Communication Delay, IEEE Trans. Instrumentation and Measurement, vol. 21, pp. 40-51, 2013.

[13] A. Kaldmäe, C.H. Moog, C. Califano, Towards integrability for nonlinear time-delay systems, MICNON 2015, St. Petersburg, Russia, IFACPapersOnLine, vol. 48, pp.900-905, 2015.

[14] A. Kaldmäe, C. Califano, C.H. Moog, Integrability for nonlinear timedelay systems, IEEE Trans. Automat. Control, vol.61 (7),pp. , 2016.

[15] J. Kim, P.H. Chang, H.S. Park, Two-Channel Transparency-Optimized Control Architectures in Bilateral Teleoperation With Time Delay, IEEE Trans. Control Systems Tech., vol. 62, pp. 2943-2953, 2013.

[16] M. Krstic, Delay Compensation for Nonlinear, Adaptive, and PDE Systems, Birkhaüser, Boston, 2009.

[17] S.J. Li, C.H. Moog, C. Califano, Characterization of accessibility for a class of nonlinear time-delay systems, Proc. of 50th IEEE CDC and ECC, Orlando, USA, pp. 1068-1073 2011.

[18] L. A. Márquez-Martínez, Note sur l'accessibilité des systèmes non linéaires à retards, Comptes Rendus de l'Académie des Sciences-Series I - Mathematics, vol. 329, pp. 545-550, 1999.

[19] L.A. Márquez-Martínez, Analyse et commande de systèmes non linéaires à retards, $\mathrm{PhD}$ thesis, Université de Nantes / Ecole Centrale de Nantes, Nantes, France, 2000.

[20] L. A. Márquez-Martínez, C.H. Moog, New insights on the analysis of nonlinear time-delay systems: Application to the triangular equivalence, Systems \& Control Letters, vol. 56, pp. 133-140, 2007.

[21] R. Murray and S. Sastry, Nonholonomic motion planning: Steering using sinusoids, IEEE Trans. Automat. Control, vol. 38, pp. 700-716, 1993.

[22] T. Oguchi, A. Watanabe and T. Nakamizo, Input-output linearization of retarded non-linear systems by using an extension of Lie derivative, Int. Journal of Control, vol. 75, pp. 582-590, 2002.

[23] G. Sallet, Claude Lobry : un mathématicien militant, Proc. of 2007 International Conference in Honor of Claude Lobry, ARIMA, vol. 9, pp. 5-13, 2008

[24] O. J. Sørdalen, Conversion of the kinematics of a car with $n$ trailers into a chained form, Proc. of 1993 International Conf. Robotics and Automation, Atlanta, USA, pp. 382-387, 1993.

[25] Timmer, J., Müller, T., I. Swameye, O. Sandra and U. Klingmüller, Modeling the nonlinear dynamics of cellular signal transduction. International Journal of Bifurcation and Chaos, vol. 14, pp. 2069-2079, 2004.

[26] X. Xia, L.A. Márquez-Martínez, P. Zagalak and C.H. Moog, Analysis of nonlinear time-delay systems using modules over non-commutative rings, Automatica, vol. 38, pp. 1549-1555, 2002.

[27] G. Zheng, J.P. Barbot, D. Boutat, T. Floquet and J.P. Richard, On observation of time-delay systems with unknown inputs, IEEE Trans. aut. Contr., vol. 56, pp. 1973-1978, 2011.

\section{APPENDIX: PROOFS AND USEFUL LEMMAS}

Proof of Proposition 1. Assume $j>0$. From the expression of $\left[\mathbf{R}_{q}(\mathbf{x}, \epsilon), r_{i}(\mathbf{x}, \delta)\right]$ one gets that

$$
\begin{aligned}
& \frac{\partial\left[\mathbf{R}_{q}(\mathbf{x}, \epsilon), r_{i}(\mathbf{x}, \delta)\right]}{\partial \epsilon\left(s_{q}-j\right)}=\sum_{\ell=-s_{q}}^{s}\left[r_{q}^{\ell+j}, r_{i}^{\ell}\right]_{E_{0}} \delta^{\ell+s_{q}} \\
& \frac{\partial\left[\mathbf{R}_{i}(\mathbf{x}, \epsilon), r_{q}(\mathbf{x}, \delta)\right]}{\partial \epsilon\left(s_{i}+j\right)}=\sum_{\ell=-s_{i}}^{s+j}\left[r_{i}^{\ell-j}, r_{q}^{\ell}\right]_{E_{0}} \delta^{\ell+s_{i}}
\end{aligned}
$$


If $s_{i} \geq s_{q},\left[r_{q}^{\ell}, r_{i}^{\ell-j}\right]_{E_{0}}=0$ for $\ell \in\left[-s_{i},-s_{q}+j\right)$, which proves (8) with $i=2$ and $q=1$. If $s_{i} \leq s_{q}$ then (8) follows, with $i=1$ and $q=2$, by considering that for $j<s_{q}-s_{i}$, $\left[r_{q}^{\ell+j}, r_{i}^{\ell}\right]_{E_{0}}=0$ for $\ell \in\left[-s_{q},-s_{i}-j\right)$, while for $s_{i}+j \geq s_{q}$, then $\left[r_{i}^{\ell-j}, r_{q}^{\ell}\right]_{E_{0}}=0$ for $\ell \in\left[-s_{i},-s_{q}+j\right)$. The case $j<0$ can be recovered in the same way. $\triangleleft$

Proof of Proposition 2. Let $\bar{r}_{i}\left(\mathbf{x}_{\left[\bar{s}_{i}, s\right]}, \delta\right)=$ $r_{i}\left(\mathbf{x}_{\left[s_{i}, s\right]}, \delta\right) \beta_{i}\left(\mathbf{x}_{\left[s_{i}, s\right]}, \delta\right), i=1,2$. Since $s_{1} \geq \bar{s}_{1}$ and $\overline{\mathbf{R}}_{1}(\mathbf{x}, \epsilon)=\sum_{j=0}^{s} r_{1}^{j}(\mathbf{x}) \beta_{1}(\mathbf{x}(-j), \epsilon(-j))$,

$$
\begin{aligned}
& {\left[\overline{\mathbf{R}}_{1}(\mathbf{x}, \epsilon), \bar{r}_{2}(\mathbf{x}, \delta)\right]=} {\left[\overline{\mathbf{R}}_{1}(\mathbf{x}, \epsilon), r_{2}(\mathbf{x}, \delta)\right] \beta_{2}\left(\mathbf{x}\left(\bar{s}_{1}\right), \delta\right) } \\
&+\left.r_{2}(\mathbf{x}, \delta) \dot{\beta}_{2}\right|_{\dot{x}_{[0]}=\overline{\mathbf{R}}_{1}(\mathbf{x}, \epsilon)} \delta^{\bar{s}_{1}} \\
& {\left[\overline{\mathbf{R}}_{1}(\mathbf{x}, \epsilon), r_{2}(\mathbf{x}, \delta)\right]=}\left.\dot{r}_{2}(\mathbf{x}, \delta)\right|_{\dot{x}_{[0]}=\overline{\mathbf{R}}_{1}(\mathbf{x}, \epsilon)} \delta^{\bar{s}_{1}} \\
&-\sum_{k=0}^{\bar{s}_{1}+s} \sum_{j=0}^{s} \beta_{1}(\mathbf{x}(-j), \epsilon(-j)) \frac{\partial r_{1}^{j}(\mathbf{x})}{\partial \mathbf{x}_{[0]}\left(\bar{s}_{1}-k\right)} \delta^{k} r_{2}\left(\mathbf{x}\left(\bar{s}_{1}\right), \delta\right) \\
&-\sum_{k=0}^{\bar{s}_{1}+s} \sum_{j=0}^{s} r_{1}^{j}(\mathbf{x}) \frac{\partial\left(\beta_{1}(\mathbf{x}(-j), \epsilon(-j))\right)}{\partial \mathbf{x}_{[0]}\left(\bar{s}_{1}-k\right)} \delta^{k} r_{2}\left(\mathbf{x}\left(\bar{s}_{1}\right), \delta\right)
\end{aligned}
$$

so that (9) follows by noting that

$$
\begin{array}{r}
{\left[\overline{\mathbf{R}}_{1}(\mathbf{x}, \epsilon), r_{2}(\mathbf{x}, \delta)\right] \delta^{s_{1}-\bar{s}_{1}}=\left.\left[\mathbf{R}_{1}(\mathbf{x}, \epsilon), r_{2}(\mathbf{x}, \delta)\right]\right|_{\bar{\epsilon}=\beta_{1}(\mathbf{x}, \epsilon)}} \\
-r_{1}(\mathbf{x}, \delta) \sum_{k=0}^{s+s_{1}} \frac{\partial \beta_{1}(\mathbf{x}, \epsilon)}{\partial \mathbf{x}_{[0]}\left(s_{1}-k\right)} \delta^{k} r_{2}\left(\mathbf{x}\left(s_{1}\right), \delta\right) .
\end{array}
$$

$\triangleleft$

Proposition 7: Given $\hat{\tau}_{1}\left(\mathbf{x}_{[p, s]}, \delta\right)=\sum_{\ell=0}^{s} \hat{\tau}_{1}^{\ell}\left(\mathbf{x}_{[p, s]}\right) \delta^{\ell}$ and the dynamics (14), let $g_{\mu, j}(\mathbf{x}, 0, \delta)=a d_{\bar{F}(\mathbf{x}, 0,1)}^{\mu-1} g_{1 j}(\mathbf{x}, \delta)$. Then for any $i \geq 1, \hat{\tau}_{i}(\mathbf{x}, \mathbf{u}, \delta)=a d_{\bar{F}(\mathbf{x}, \mathbf{u}, 1)}^{i-1} \hat{\tau}_{1}(\mathbf{x}, \delta)$, can be computed by considering $F_{0 i}(\mathbf{x}, \delta)=\sum_{l=0}^{i s+p} F(\mathbf{x}) \delta^{l}$, so that $F_{0 i}^{l}=F(\mathbf{x})$. More precisely the following relations hold true:

i.) Given $\tau_{1}=\hat{\tau}_{1}\left(\mathbf{x}_{[p, s]}, \delta\right), \alpha=\alpha(\mathbf{x}, \mathbf{u}, \delta)$,

$$
a d_{\bar{F}(\mathbf{x}, \mathbf{u}, 1)}^{k}\left(\hat{\tau}_{1}(\mathbf{x}, \delta) \alpha\right)=\sum_{j=0}^{k}\left(\begin{array}{c}
k \\
j
\end{array}\right) \hat{\tau}_{k-j+1}(\mathbf{x}, \mathbf{u}, \delta) \alpha^{(j)}
$$

ii.) $\hat{\tau}_{i}(\mathbf{x}, \mathbf{u}, \delta)=a d_{\bar{F}(\mathbf{x}, \mathbf{u}, 1)}^{i-1} \hat{\tau}_{1}(\mathbf{x}, \delta)$ is given by

$$
\begin{gathered}
a d_{\bar{F}(\mathbf{x}, \mathbf{u}, 1)}^{i-1} \hat{\tau}_{1}(\mathbf{x}, \delta)=\hat{\tau}_{i, 0}(\mathbf{x}, \delta)+\sum_{j=1}^{m} \sum_{q=0}^{i-2} \sum_{\mu=1}^{i-1-q} \\
\sum_{k=-p-i s}^{p+i s} \sum_{\ell=0}^{i s}\left(\begin{array}{c}
i-1 \\
\mu+q
\end{array}\right) c_{\mu}^{q}\left[g_{\mu, j}^{k+\ell}(0), \hat{\tau}_{i-\mu-q}^{\ell}\right]_{E_{0}} \delta^{\ell} u_{j,[0]}^{(q)}(-k)(35) \\
+m_{i}\left(\mathbf{x}, \mathbf{u}^{[i-3]}, \delta\right)
\end{gathered}
$$

where $c_{\mu}^{0}=c_{1}^{q}=1$, and for $\mu>1, q>0, c_{\mu}^{q}=$ $c_{\mu-1}^{q}+c_{\mu}^{q-1}$,

$$
\begin{aligned}
\hat{\tau}_{i, 0}(\mathbf{x}, \delta) & =a d_{\bar{F}(\mathbf{x}, 0,1)}^{i-1} \hat{\tau}_{1}(\mathbf{x}, \delta) \\
& =\sum_{\ell}\left[F_{0 i}^{i s} \cdots,\left[F_{0 i}^{i s}, \tau_{1}^{\ell}\right]_{E_{i s}}\right]_{E_{0}} \delta^{\ell}
\end{aligned}
$$

and $m_{i}\left(\mathbf{x}, \mathbf{u}^{[i-3]}, \delta\right)$ is given by the linear combination, through real coefficients, of terms of the form

$\sum_{\ell}\left[g_{\mu_{1}, j_{1}}^{i_{1}+\ell}(0), \cdots,\left[g_{\mu_{\nu}, j_{\nu}}^{i_{\nu}+\ell}(0), \tau_{i-q}^{\ell}\right]_{E_{i s}}\right]_{E_{0}} \delta^{\ell} \prod_{\mu=1}^{\nu} u_{j_{\mu},[0]}^{\left(\ell_{\mu}\right)}\left(-i_{\mu}\right)$. where $\nu \in[2, i-1], j_{\nu} \in[1, m], q=\sum_{k=1}^{\nu} \ell_{k}+\mu_{k} \leq i-1$.

Proof. i) Let us preliminary note that by definition

$$
\begin{gathered}
a d_{\bar{F}(\cdot, \mathbf{u}, 1)}\left(\hat{\tau}_{1}(\mathbf{x}, \delta) \alpha(\mathbf{x}, \mathbf{u}, \delta)\right)=\frac{d}{d t}\left(\hat{\tau}_{1} \alpha\right)-f(\mathbf{x}, \mathbf{u}, \delta) \hat{\tau}_{1} \alpha \\
=\hat{\tau}_{2}(\mathbf{x}, \mathbf{u}, \delta) \alpha(\mathbf{x}, \mathbf{u}, \delta)+\hat{\tau}_{1}(\mathbf{x}, \delta) \dot{\alpha}
\end{gathered}
$$

The proof is iterative. Assume that it is true for $k-1$, we will verify it for $k$. In fact, using (37), and dropping for simplicity the dependence from $\mathbf{x}, \mathbf{u}$ and $\delta$,

$$
\begin{aligned}
a d_{\bar{F}(\cdot, \mathbf{u}, 1)}^{k}\left(\hat{\tau}_{1} \alpha\right) & =a d_{\bar{F}(\cdot, \mathbf{u}, 1)}\left(\sum_{j=0}^{k-1}\left(\begin{array}{c}
k-1 \\
j
\end{array}\right) \hat{\tau}_{k-j} \alpha^{(j)}\right) \\
& =\sum_{j=0}^{k-1}\left(\begin{array}{c}
k-1 \\
j
\end{array}\right)\left(\hat{\tau}_{k-j+1} \alpha^{(j)}+\hat{\tau}_{k-j} \dot{\alpha}^{(j)}\right)
\end{aligned}
$$

which proves the results since $\left(\left(\begin{array}{c}k-1 \\ j\end{array}\right)+\left(\begin{array}{c}k-1 \\ j-1\end{array}\right)\right) \alpha^{(j)}=$ $\left(\begin{array}{c}k \\ j\end{array}\right) \alpha^{(j)}$, for $j \in[1, k-1]$.

ii.) According to (7) with $r_{1}(\mathbf{x}, \epsilon)=F\left(\mathbf{x}_{[s]}\right) \epsilon(0)$,

$$
a d_{\bar{F}(\mathbf{x}, 0,1)} \hat{\tau}_{1}\left(\mathbf{x}_{[p, s]}, \delta\right)=\sum_{j=-2 s}^{2 s+p} \sum_{\ell=0}^{2 s}\left[r_{1}^{\ell-j}, \hat{\tau}_{1}^{\ell}\right]_{E_{0}} \delta^{\ell}
$$

Since only $r_{1}^{0}=F(\mathbf{x}) \neq 0$, then introducing $F_{0 i}(\mathbf{x}, \delta)=$ $\sum_{l=0}^{i s+p} F(\mathbf{x}) \delta^{l}$, one gets that $\sum_{j=-2 s}^{2 s+p}\left[r_{1}^{\ell-j}, \hat{\tau}_{1}^{\ell}\right]_{E_{0}}=$ $\left[F_{0 i}^{i s}, \hat{\tau}_{1}^{l}\right]_{E_{0}}$. Iteratively (36) follows.

Set now $r_{2}(x, \epsilon)=\sum_{\nu=1}^{m} \sum_{j=0}^{s} g_{1, \nu}^{j} u_{\nu,[0]}(-j) \epsilon(0)$ and let $a d_{\bar{F}(\mathbf{x}, 0,1)}^{i-1} \hat{\tau}_{1}=\hat{\tau}_{i, 0}\left(\mathbf{x}_{[p, i s]}, \delta\right)$. Then

$$
\begin{aligned}
& a d_{\bar{F}(\mathbf{x}, \mathbf{u}, 1)} \tau_{1}(\mathbf{x}, \delta)= \\
& =\hat{\tau}_{2,0}+\sum_{\nu=1}^{m} \sum_{\ell=0}^{2 s} \sum_{j=-p}^{2 s}\left[g_{1 \nu}^{j}, \hat{\tau}_{1}^{\ell}\right]_{E_{0}} \delta^{\ell} u_{\nu,[0]}(-j+\ell) \\
& =\hat{\tau}_{2,0}+\sum_{\nu=1}^{m} \sum_{\ell=0}^{2 s} \sum_{j=-p-\ell}^{2 s-\ell}\left[g_{1 \nu}^{j+\ell}, \hat{\tau}_{1}^{\ell}\right]_{E_{0}} \delta^{\ell} u_{\nu,[0]}(-j)
\end{aligned}
$$

With the introduced notation, for some fixed $j$ and $\nu$ :

$$
\begin{gathered}
a d_{\bar{F}(\mathbf{x}, 0,1)} \sum_{\ell=0}^{2 s}\left[g_{1 \nu}^{j+\ell}, \hat{\tau}_{1}^{\ell}\right]_{E_{0}} \delta^{\ell}= \\
\sum_{\ell=0}^{3 s}\left[g_{2, \nu}^{j+\ell}(0), \hat{\tau}_{1}^{\ell}\right]_{E_{0}} \delta^{\ell}+\sum_{\ell=0}^{3 s}\left[g_{1 \nu}^{j+\ell}, \hat{\tau}_{2,0}^{\ell}\right]_{E_{0}} \delta^{\ell}
\end{gathered}
$$

The proof of ii) is by induction. Assume that the expression of $\tau_{k}(\mathbf{x}, \mathbf{u}, \delta)=a d_{\bar{F}(\cdot, u, 1)}^{k-1} \tau_{1}(\mathbf{x}, \delta)$ is given by ii) for $k=i-1$, then we will prove that is verified also for $k=i$. In fact, through standard computation one gets

$$
\begin{gathered}
\tau_{i}(\mathbf{x}, \mathbf{u}, \delta)=a d_{F(\mathbf{x}, \mathbf{u}, 1)} \tau_{i-1}(\mathbf{x}, \mathbf{u}, \delta)=a d_{F(\mathbf{x}, \mathbf{u}, 1)} \hat{\tau}_{i-1,0}(\mathbf{x}, \delta)+ \\
\sum\left(\begin{array}{c}
i-2 \\
\mu+q
\end{array}\right) c_{\mu}^{q} a d_{F(\mathbf{x}, \mathbf{u}, 1)}\left(\left[g_{\mu, j}^{k+\ell}(0), \hat{\tau}_{i-1-\mu-q}^{\ell}\right]_{E_{0}} \delta^{\ell}\right) u_{j,[0]}^{(q)}(-k)+ \\
\sum\left(\begin{array}{c}
i-2 \\
\mu+q
\end{array}\right) c_{\mu}^{q}\left[g_{\mu, j}^{k+\ell}(0), \hat{\tau}_{i-1-\mu-q}^{\ell}\right]_{E_{0}} \delta^{\ell} u_{j,[0]}^{(q+1)}(-k) \\
+a d_{F(\mathbf{x}, \mathbf{u}, 1)} m_{i-1}(\mathbf{x}, \mathbf{u}, \delta)
\end{gathered}
$$

where the summations are meant with respect to the indices $(\mu, k, \ell, j, q)$. The result follows after standard computations 
by noting that

$$
\begin{gathered}
\operatorname{ad}_{F(\mathbf{x}, \mathbf{u}, 1)}\left(\sum_{\ell}\left[g_{\mu, j}^{k+\ell}(0), \hat{\tau}_{i-1-\mu-q}^{\ell}\right]_{E_{0}} \delta^{\ell}\right) u_{j,[0]}^{(q)}(-k)= \\
\operatorname{ad}_{F(\mathbf{x}, 0,1)}\left(\sum_{\ell}\left[g_{\mu, j}^{k+\ell}(0), \hat{\tau}_{i-1-\mu-q}^{\ell}\right]_{E_{0}} \delta^{\ell}\right) u_{j,[0]}^{(q)}(-k) \\
+ \text { terms in } m_{i}(., \delta),
\end{gathered}
$$

using equation (38), by recalling that $c_{\mu}^{q}=c_{\mu-1}^{q}+$ $c_{\mu}^{q-1}$ and that $\left(\begin{array}{c}i-2 \\ k-1\end{array}\right)+\left(\begin{array}{c}i-2 \\ k\end{array}\right)=\left(\begin{array}{c}i-1 \\ k\end{array}\right)$, and noting that $a d_{F(\mathbf{x}, \mathbf{u}, 1)} m_{i-1}(\mathbf{x}, \mathbf{u}, \delta)$ leads to terms in $m_{i}(\mathbf{x}, \mathbf{u}, \delta), \triangleleft$

The following results hold true.

Lemma 2: Consider the distribution $\Delta_{i}$ defined by (13), and let $\rho_{i}=\operatorname{dim}\left(\Delta_{1}\right)$ with $\rho_{-1}=n s$. Then

(i) If $d \lambda(\mathbf{x})$ is such that $\operatorname{span}\{d \lambda(\mathbf{x})\}=\bar{\Delta}_{i-1}^{\perp}$, then $\operatorname{span}\{d \lambda(\mathbf{x}), d \lambda(\mathbf{x}(-1))\} \subset \bar{\Delta}_{i}^{\perp}$.

(ii) A canonical basis for $\bar{\Delta}_{i}^{\perp}$ is defined for $i \geq 0$ as follows. Pick $d \lambda_{0}\left(\mathbf{x}_{[0]}\right)$ such that $\operatorname{span}\left\{d \lambda_{0}\left(\mathbf{x}_{[0]}\right)\right\}=\bar{\Delta}_{0}^{\perp}$, with $\operatorname{rank} d\left(\lambda_{0}\right)=\mu_{0}=\rho_{0}-\rho_{-1}$.

At step $\ell \leq i$ pick $d \lambda_{\ell}\left(\mathbf{x}_{[\ell]}\right)$ such that $\operatorname{span}\left\{d \lambda_{k}\left(\mathbf{x}_{[k]}(-j)\right), k \in[0, \ell], j \in[0, \ell-k]\right\}=\bar{\Delta}_{\ell}^{\perp}$ and $d \lambda_{\ell}\left(\mathbf{x}_{[\ell]}\right) \notin \bar{\Delta}_{\ell-1}^{\perp}$, with $\operatorname{rank} d\left(\lambda_{i}\right)=\mu_{i}=$ $\rho_{i}-2 \rho_{i-1}+\rho_{i-2}$.

Proof. The proof of (i) can be easily carried out by considering, for $i \geq 1$, on $\mathbb{R}^{n(\bar{s}+s+i+1)}, \Delta_{i-1}^{(j)} \subset$ $\operatorname{span}\left\{\frac{\partial}{\partial \mathbf{x}_{[0]}(\bar{s})}, \cdots, \frac{\partial}{\partial \mathbf{x}_{[0]}(-i-s)}\right\}, j=1,2$, defined as

$$
\begin{aligned}
& \Delta_{i-1}^{(1)}=\left(\begin{array}{c}
\Delta_{i-1} \\
0
\end{array}\right)+\operatorname{span}\left\{\frac{\partial}{\partial \mathbf{x}_{[0]}(-i-s)}\right\}, \\
& \Delta_{i-1}^{(2)}=\left(\begin{array}{c}
0 \\
\Delta_{i-1}(-1)
\end{array}\right)+\operatorname{span}\left\{\frac{\partial}{\partial \mathbf{x}_{[0]}(\bar{s})}\right\}
\end{aligned}
$$

By construction $\Delta_{i} \subset \Delta_{i-1}^{(j)}, j=1,2$ so that $\bar{\Delta}_{i} \subset \bar{\Delta}_{i-1}^{(j)}$, $j=1,2$ and consequently $\bar{\Delta}_{i}^{\perp} \supset\left(\bar{\Delta}_{i-1}^{(1)}\right)^{\perp}=\bar{\Delta}_{i-1}^{\perp}$ and $\bar{\Delta}_{i}^{\perp} \supset$ $\left(\bar{\Delta}_{i-1}^{(2)}\right)^{\perp}=\bar{\Delta}_{i-1}^{\perp}(-1)$. It follows that any $d \varphi\left(\mathbf{x}_{[i-1]}\right) \in \bar{\Delta}_{i-1}^{\perp}$ satisfies also $d \varphi\left(\mathbf{x}_{[i-1]}\right) \in \bar{\Delta}_{i}^{\perp}, d \varphi\left(\mathbf{x}_{[i-1]}\right)(-1) \in \bar{\Delta}_{i}^{\perp}$.

Due to the previous result the proof of (ii) is immediate. $\triangleleft$

\section{A. Basis Completion}

Lemma 3: Given $n-k$ independent functions $\lambda_{i}\left(\mathbf{x}_{[\alpha]}\right)$, $i \in[1, n-k]$, such that $\operatorname{span}_{\mathcal{K}(\delta]}\left\{d \lambda_{1}, \cdots, d \lambda_{n-k}\right\}$ is closed and its right annihilator is causal, there exists a $d \theta_{1}\left(\mathbf{x}_{[\bar{\alpha}]}\right)$ independent of the $d \lambda_{i}\left(\mathbf{x}_{[\alpha]}\right)$ 's $i \in[1, n-k]$ over $\mathcal{K}(\delta]$ and such that $\operatorname{span}_{\mathcal{K}(\delta]}\left\{d \lambda_{1}, \cdots, d \lambda_{n-k}, d \theta_{1}\right\}$ is closed and its right annihilator is causal.

Proof. By assumption $\operatorname{span}_{\mathcal{K}(\delta]}\left\{d \lambda_{1}, \cdots, d \lambda_{n-k}\right\}$ is closed and its right annihilator is causal, so that according to Theorem 13 in [20], it is possible to compute, using Smith decomposition, $\omega^{T}(\mathbf{x}, \delta)=\left(\omega_{1}^{T}(\mathbf{x}, \delta), \cdots, \omega_{k}^{T}(\mathbf{x}, \delta)\right)^{T}$ causal such that setting $d \lambda(\mathbf{x})=\Lambda(\mathbf{x}, \delta) d \mathbf{x}_{[0]}$ then

$$
\left(\begin{array}{c}
\Lambda(\mathbf{x}, \delta) \\
\omega(\mathbf{x}, \delta)
\end{array}\right)=T(\mathbf{x}, \delta)
$$

is a unimodular causal matrix. Let $T^{-1}(\mathbf{x}, \delta)=$ $\left(p_{1}(\mathbf{x}, \delta), \cdots, p_{n}(\mathbf{x}, \delta)\right)$, set $\bar{p}^{i}=\left[p_{n-k+1}^{i}, \cdots, p_{n}^{i}\right]$ and denote by $\bar{p}(\mathbf{x}, \delta)=\sum_{i=0}^{s} \bar{p}^{i} \delta^{i}$. Let, without loss of generality, $\bar{p}(\mathbf{x}, \delta)$ be characterized by the minimal degree $s$ which can be attained by post multiplication by a unimodular causal matrix. In fact, if $\bar{p}(\mathbf{x}, \delta)=\hat{p}(\mathbf{x}, \delta) q(\mathbf{x}, \delta)$, with $\operatorname{deg}(\hat{p}(\mathbf{x}, \delta))<\operatorname{deg}(\bar{p}(\mathbf{x}, \delta))$ and $q(\mathbf{x}, \delta)$ unimodular, then it would be sufficient to take as new basis $\left(\begin{array}{c}\Lambda(\mathbf{x}) \\ \bar{\omega}(\mathbf{x}, \delta)\end{array}\right)$ where $\bar{\omega}(\mathbf{x}, \delta)=q(\mathbf{x}, \delta) \omega(\mathbf{x}, \delta)$. In fact, one has that

$$
\begin{aligned}
\left(\begin{array}{c}
\Lambda(\mathbf{x}) \\
\bar{\omega}(\mathbf{x}, \delta)
\end{array}\right) \hat{p}(\mathbf{x}, \delta) & =\left(\begin{array}{cc}
I & 0 \\
0 & q(\mathbf{x}, \delta)
\end{array}\right)\left(\begin{array}{c}
\Lambda(\mathbf{x}) \\
\omega(\mathbf{x}, \delta)
\end{array}\right) \bar{p}(\mathbf{x}, \delta) q^{-1}(\mathbf{x}, \delta) \\
& =\left(\begin{array}{cc}
I & 0 \\
0 & q(\mathbf{x}, \delta)
\end{array}\right)\left(\begin{array}{c}
0 \\
q^{-1}(\mathbf{x}, \delta)
\end{array}\right)=\left(\begin{array}{c}
0 \\
I
\end{array}\right) .
\end{aligned}
$$

Consider now for $\ell \geq 0$, the sequence of distributions $\Delta_{\ell}=\Delta_{[0, \ell+s]}$ defined as

$$
\Delta_{\ell}=\operatorname{span}\left(\begin{array}{cccccc}
\bar{p}^{0} & \cdots & \bar{p}^{s} & 0 & & 0 \\
0 & \ddots & & \ddots & \ddots & \\
& \ddots & \bar{p}^{0}(-\ell) & \cdots & \bar{p}^{s}(-\ell) & 0 \\
0 & \cdots & 0 & \cdots & 0 & I_{n s}
\end{array}\right),
$$

and denote by $\Delta_{\ell, 0}$, with $\ell \geq 0$, the distribution obtained from $\Delta_{\ell}$ by eliminating the first $k$ columns, that is

$\Delta_{\ell, 0}=\operatorname{span}\left(\begin{array}{ccccccc}\bar{p}^{1} & \cdots & \bar{p}^{s} & 0 & \cdots & & \\ \bar{p}^{0}(-1) & \cdots & & \bar{p}^{s}(-1) & 0 & & \\ 0 & \ddots & \vdots & & \ddots & & \\ & \cdots & & & & \bar{p}^{s}(-\ell) & 0 \\ 0 & \cdots & 0 & \cdots & & 0 & I_{n s}\end{array}\right)$.

By assumption

$$
\begin{aligned}
\Lambda_{i}(\mathbf{x}, \delta) \bar{p}(\mathbf{x}, \delta) & =0, \quad i \in[1, n-k] \\
\omega_{i}(\mathbf{x}, \delta) \bar{p}(\mathbf{x}, \delta) & =[0 \cdots 1 \cdots 0], \quad i \in[1, k] .
\end{aligned}
$$

Let $j$ be the maximum delay in $\Lambda_{i}(\mathbf{x}, \delta) i \in[1, n-k]$ and $\omega_{i}(\mathbf{x}, \delta)=\sum_{l=0}^{j} \omega_{i l} \delta^{l}, i \in[1, k]$. Then we have that $d \lambda_{i} \bar{\Delta}_{j}=0$, whereas $\left[\omega_{0}, \omega_{1}, \cdots, \omega_{j}\right] \Delta_{j} \neq 0$. More precisely by construction $\omega_{0} \bar{p}^{0}=I$, whereas $\left[\omega_{0}, \omega_{1}, \cdots, \omega_{j}\right] \Delta_{j 0}=0$.

We first prove that there exists a $d \theta_{1}\left(\mathbf{x}_{[\bar{\alpha}]}\right) \in \bar{\Delta}_{j 0}^{\perp}$, with $\bar{\alpha} \leq j$, which is independent of the $d \lambda_{i}$ 's $i \in[1, n-k]$ over $\mathcal{K}(\delta]$. Assume that it is not true, then there must exist $k$ extended Lie brackets such that $\left[p_{l_{q}}^{i_{q}}, p_{\nu_{q}}^{k_{q}}\right]_{E_{k_{q}}}=\tau_{0 q}(\mathbf{x})+$ $\bar{p}^{0}(\mathbf{x}) \alpha_{0 q}(\mathbf{x}) \frac{\partial}{\partial \mathbf{x}_{[0]}}$, (with $i_{q} \geq k_{q} \geq 1$ ) where $\tau_{0 q}(\mathbf{x})=$ $\sum_{l=0}^{p} \tau_{q}^{p-l}(-l) \frac{\partial}{\partial \mathbf{x}_{[0]}(-l)} \in \Delta_{j 0}, q \in[1, k]$. Furthermore the $k \times k$ matrix $\alpha_{0}=\left[\alpha_{01}, \cdots, \alpha_{0 k}\right]$ is of full rank.

Consider now $\Delta_{j+1}$ and correspondingly $\Delta_{j+1,0}$. Accordingly, consider the $k$ extended Lie brackets $\left[p_{l_{q}}^{i_{q}+1}, p_{\nu_{q}}^{k_{q}+1}\right]_{E_{k_{q}+1}}, q \in[1, k]$. By construction

$$
\left[p_{l_{q}}^{i_{q}+1}, p_{\nu_{q}}^{k_{q}+1}\right]_{E_{k_{q}+1}}=\tau_{1 q}(\mathbf{x})+\left(\bar{p}^{0}(\mathbf{x})\right)^{T} \alpha_{1 q}(\mathbf{x}) \frac{\partial}{\partial \mathbf{x}_{[0]}}
$$

$+\left(\left(\bar{p}^{1}(\mathbf{x})\right)^{T} \frac{\partial}{\partial \mathbf{x}_{[0]}}+\left(\bar{p}^{0}(\mathbf{x}(-1))\right)^{T} \frac{\partial}{\partial \mathbf{x}_{[0]}(-1)}\right) \alpha_{0 q}(\mathbf{x}(-1))$

where $\tau_{1 q}(\mathbf{x})=\sum_{l=0}^{p+1}\left(\tau_{q}^{p+1-l}(\mathbf{x}(-l))\right)^{T} \frac{\partial}{\partial \mathbf{x}_{[0]}(-l)} \in \Delta_{j+1,0}$. 
In fact, if (42) were not satisfied then

$$
\begin{aligned}
& {\left[p_{l_{q}}^{i_{q}+1}, p_{\nu_{q}}^{k_{q}+1}\right]_{E_{k_{q}+1}}-\tau_{1 q}(\mathbf{x})} \\
& \quad-\left(\left(\bar{p}^{1}(\mathbf{x})\right)^{T} \frac{\partial}{\partial \mathbf{x}_{[0]}}+\left(\bar{p}^{0}(\mathbf{x}(-1))\right)^{T} \frac{\partial}{\partial \mathbf{x}_{[0]}(-1)}\right) \alpha_{0 q}(\mathbf{x}(-1)) \\
& \quad=(r(\mathbf{x}))^{T} \frac{\partial}{\partial \mathbf{x}_{[0]}} \notin \operatorname{span}\left\{\left(\bar{p}^{0}\right)^{T} \frac{\partial}{\partial \mathbf{x}_{[0]}}\right\} \\
& \text { against the assumption of } n-k \text { independent exact differentials } \\
& \text { in the left kernel of } \bar{p}(\mathbf{x}, \delta) \text {. Iterating the reasoning one gets } \\
& \text { that for any } \beta \geq 0, \\
& \qquad\left[p_{l_{q}}^{i_{q}+\beta}, p_{\nu_{q}}^{k_{q}+\beta}\right]_{E_{k_{q}+\beta}}=\tau_{\beta q}(\mathbf{x}) \\
& \quad+\sum_{\gamma=0}^{\beta} \sum_{l=0}^{\beta-\gamma}\left(\bar{p}^{\beta-\gamma-l}(\mathbf{x}(-l))\right)^{T} \alpha_{\gamma, q}(\mathbf{x}(-\beta+\gamma)) \frac{\partial}{\partial \mathbf{x}_{[0]}(-l)}
\end{aligned}
$$$$
-\left(\left(\bar{p}^{1}(\mathbf{x})\right)^{T} \frac{\partial}{\partial \mathbf{x}_{[0]}}+\left(\bar{p}^{0}(\mathbf{x}(-1))\right)^{T} \frac{\partial}{\partial \mathbf{x}_{[0]}(-1)}\right) \alpha_{0 q}(\mathbf{x}(-1)) d \theta_{1}\left(\mathbf{x}_{[\bar{\alpha}]}\right)=\sum_{j=1}^{n-k} \gamma_{j}(\mathbf{x}, \delta) d \lambda_{j}\left(\mathbf{x}_{[\alpha]}\right)+\sum_{j=1}^{k} \nu_{j}(\mathbf{x}, \delta) \omega_{j}(\mathbf{x}, \delta) d \mathbf{x}_{[0]}
$$

and $\tau_{\beta, q}(\mathbf{x})=\sum_{l=0}^{p+\beta}\left(\tau_{q}^{p+\beta-l}(\mathbf{x}(-l))\right)^{T} \frac{\partial}{\partial \mathbf{x}_{[0]}(-l)} \in \Delta_{j+\beta, 0}$. Since by assumption $\bar{p}^{l}=0$ for $l>s$, one gets that as soon as $k_{q}+\beta \geq s+1,\left[p_{l_{q}}^{i_{q}+\beta}, p^{k_{q}+\beta} \nu_{q}\right]_{E_{0}}=0 \forall l_{q}, \nu_{q} \in[n-k+1, n]$. Furthermore, since $\tau_{0, q} \in \Delta_{j 0}$, then necessarily there exists an index $l \leq s$ such that $\tau_{q}^{l+i}=0, \forall i \geq 0$. As a consequence, there exists an index $\beta \leq s$, such that for any $\theta \in[\beta, s]$, and $\forall l_{q}, \nu_{q} \in[n-k+1, n]$,

$$
0=\left[p_{l_{q}}^{i_{q}+\theta}, p_{\nu_{q}}^{k_{q}+\theta}\right]_{E_{0}}=\sum_{\gamma=0}^{\theta}\left(\bar{p}^{\theta-\gamma}(\mathbf{x})\right)^{T} \alpha_{\gamma, q}(\mathbf{x}(-\theta+\gamma)) \frac{\partial}{\partial \mathbf{x}_{[0]}}
$$

Consider now the distribution $\tilde{\Delta}_{j}$ obtained by combining linearly the columns of $\Delta_{j}$ through the matrix

$$
\alpha=\left(\begin{array}{cccc}
\alpha_{0} & \cdots & \alpha_{s+j} & 0 \\
0 & \ddots & \vdots & \vdots \\
0 & 0 & \alpha_{0}(-s-j) & 0 \\
\vdots & \cdots & 0 & I_{n s}
\end{array}\right)
$$

that is $\tilde{\Delta}_{j}=\Delta_{j} \alpha$. By construction we have that

$$
\tilde{\Delta}_{j}=\operatorname{span}\left(\begin{array}{ccccc}
\tilde{p}^{0} & \cdots & \tilde{p}^{\theta} & 0 \cdots & 0 \\
0 & \ddots & \cdots & \ddots & \vdots \\
& \tilde{p}^{0}(-j) & \cdots & \tilde{p}^{\theta}(-j) & 0 \\
0 & 0 & * & * & I_{n s}
\end{array}\right) .
$$

Since $\operatorname{span} \Delta_{j}=\operatorname{span} \tilde{\Delta}_{j}$, through the sequence of distributions $\tilde{\Delta}_{j}$, we can recover the functions $\lambda_{i}, i \in[1, n-k]$. As a consequence we get that $\Lambda_{i}(\mathbf{x}, \delta) \tilde{p}(\mathbf{x}, \delta)=0, i \in[1, n-k]$. Since the functions $\lambda_{i}\left(\mathbf{x}_{[\alpha]}\right)$ are linearly independent, there must necessarily exist a $\bar{\alpha}(\mathbf{x}, \delta)=\sum_{i=0}^{\mu} \bar{\alpha}_{i}(\mathbf{x}) \delta^{i}$ such that

$$
\tilde{p}(\mathbf{x}, \delta)=\bar{p}(\mathbf{x}, \delta) \bar{\alpha}(\mathbf{x}, \delta)=\sum_{i=0}^{\theta} \tilde{p}^{i}(\mathbf{x}) \delta^{i}
$$

where by construction $\theta<s$. This leads to a contradiction since by assumption $\bar{p}(\mathbf{x}, \delta)$ was of minimal degree obtainable through unimodular and causal transformation, and any other transformation cannot attain a smaller degree, thus proving the existence of $d \theta_{1}\left(\mathbf{x}_{[\bar{\alpha}]}\right)$, which is independent over $\mathcal{K}(\delta]$ of the $d \lambda_{i}(\mathbf{x})$ 's. Since $\operatorname{span}_{\mathcal{K}(\delta]}\left\{d \lambda_{1}, \cdots, d \lambda_{n-k}, \omega_{1} d \mathbf{x}_{[0]}, \omega_{k} d \mathbf{x}_{[0]}\right\}$ forms a basis over $\mathbb{R}^{n}$ then
Since $d \theta_{1}\left(\mathbf{x}_{[\bar{\alpha}]}\right) \Delta_{j 0}=0$, where as $d \theta_{1}\left(\mathbf{x}_{[\bar{\alpha}]}\right) \Delta_{j} \neq 0$, then

$$
\Theta\left(\mathbf{x}_{[\bar{\alpha}]}, \delta\right) \bar{p}(\mathbf{x}, \delta)=\left[\nu_{1}(\mathbf{x}, \delta), \cdots, \nu_{k}(\mathbf{x}, \delta)\right]=\Theta^{0} \bar{p}^{0} \neq 0 .
$$

which proves that $\nu_{i}(\mathbf{x}, \delta)=\nu_{i}^{0}(\mathbf{x})$. Assuming without loss of generality $\nu_{1}=\nu_{1}^{0} \neq 0$, then

$$
\left(\begin{array}{c}
d \lambda_{1} \\
\cdots \\
d \lambda_{n-k} \\
d \theta_{1} \\
\omega_{2} d \mathbf{x}_{[0]} \\
\vdots \\
\omega_{k} d \mathbf{x}_{[0]}
\end{array}\right)=\left(\begin{array}{cccc}
I & 0 & \cdots & 0 \\
\gamma & \nu_{1} & \cdots & \nu_{k} \\
0 & 0 & I & 0 \\
0 & 0 & 0 & 1
\end{array}\right)\left(\begin{array}{c}
d \lambda_{1} \\
\cdots \\
d \lambda_{n-k} \\
\omega_{1} d \mathbf{x}_{[0]} \\
\omega_{2} d \mathbf{x}_{[0]} \\
\vdots \\
\omega_{k} d \mathbf{x}_{[0]}
\end{array}\right)
$$

which shows that

$$
\left(d \lambda_{1}^{T}, \cdots, d \lambda_{n-k}^{T}, d \theta_{1}^{T},\left(\omega_{2} d \mathbf{x}_{[0]}\right)^{T}, \cdots,\left(\omega_{k} d \mathbf{x}_{[0]}\right)^{T}\right)^{T}
$$

can be taken as a new basis being linked through a unimodular causal matrix to the old basis. As a consequence $\operatorname{span}_{\mathcal{K}(\delta]}\left\{d \lambda_{1}, \cdots, d \lambda_{n-k}, d \theta_{1}\right\}$ is closed and its right annihilator is causal. $\triangleleft$

\section{B. Properties of the Left Annihilator of a Module}

Lemma 4: Consider the matrix

$$
\Gamma\left(\mathbf{x}_{[p, s]}, \delta\right)=\left(\tau_{1}\left(\mathbf{x}_{[p, s]}, \delta\right), \cdots, \tau_{j}\left(\mathbf{x}_{[p, s]}, \delta\right)\right)
$$

Let $\bar{s}=\operatorname{deg}\left(\Gamma\left(\mathbf{x}_{[p, s]}, \delta\right)\right)$. The left annihilator $\Omega\left(\mathbf{x}_{[\bar{p}, \alpha]}, \delta\right)$ satisfies the following relations

i) $\operatorname{deg}\left(\Omega\left(\mathbf{x}_{[\bar{p}, \alpha]}, \delta\right)\right) \leq j[\operatorname{deg}(\Gamma(\mathbf{x}, \delta))]$

ii) $\bar{p}, \alpha$ can be chosen to be $\alpha \leq s+\operatorname{deg}(\Omega(\mathbf{x}, \delta)), \bar{p} \leq p$.

Consequently, if $\Gamma(\mathbf{x}, \delta)$ is causal, then $\Omega(\mathbf{x}, \delta)$ is also causal.

Proof. Without loss of generality, assume that the first $j$ rows of $\Gamma\left(\mathbf{x}_{[p, s]}, \delta\right)$ are linearly independent over $\mathcal{K}(\delta]$. Then $\Omega\left(\mathbf{x}_{[\bar{p}, \alpha]}, \delta\right)$ must satisfy

$$
\Omega(\mathbf{x}, \delta) \Gamma(\mathbf{x}, \delta)=\left[\Omega_{1}(\mathbf{x}, \delta), \Omega_{2}(\mathbf{x}, \delta)\right]\left(\begin{array}{c}
\Gamma_{1}(\mathbf{x}, \delta) \\
\Gamma_{2}(\mathbf{x}, \delta)
\end{array}\right)=0
$$

where $\Gamma_{1}(\mathbf{x}, \delta)$ is a $j \times j$ full rank matrix, accordingly $\Gamma_{2}(\mathbf{x}, \delta)$ is a $(n-j) \times j$ matrix, $\Omega_{1}(\mathbf{x}, \delta)$ is a $(n-j) \times j$ matrix and $\Omega_{2}(\mathbf{x}, \delta)$ is a $(n-j) \times(n-j)$ matrix. Let $r_{\Omega_{1}}=$ $\operatorname{deg}\left(\Omega_{1}(\mathbf{x}, \delta)\right), r_{\Omega_{2}}=\operatorname{deg}\left(\Omega_{2}(\mathbf{x}, \delta)\right), r_{\Gamma_{1}}=\operatorname{deg}\left(\Gamma_{1}(\mathbf{x}, \delta)\right)$, $r_{\Gamma_{2}}=\operatorname{deg}\left(\Gamma_{2}(\mathbf{x}, \delta)\right)$. Then we have that $r_{\Omega_{1}}+r_{\Gamma_{1}}=r_{\Omega_{2}}+r_{\Gamma_{2}}$. 